\title{
THE LINEAR ARRANGEMENT OF A SERIES OF ALLELES OF ASPERGILLUS NIDULANS
}

\author{
R. H. PRITCHARD \\ Department of Genetics, University of Glasgow
}

\section{INTRODUCTION}

Received 24.vi.55

THE discovery of recombination between the two physiologically allelic mutants "Star" and " asteroid" in Drosophila melanogaster and the difference in phenotype between heterozygotes for the two mutants in cis and trans (Lewis, 1945) provided support for the conclusion of Raffel and Muller (1940), based on their analysis of the "scute" region of Drosophila, that definitions of the gene based on the tests of separate mutation, recombination and breakage, and the physiological test of non-allelism need not be coextensive. The number of reported cases of a similar nature from several organisms has now become so large as to lead to the suggestion (Pontecorvo, I954, I955) that the ability to recombine is a common rather than exceptional property of physiologically allelic mutants.

Two types of working model have been considered to account for this type of position effect (or "Lewis effect", see Pontecorvo, 1955). One (Raffel and Muller, I940 ; Muller, r947; Pontecorvo, $\mathrm{r} 952 a, b)$ is that the unit of function, the gene, has several sites able to mutate independently and between which crossing over can occur. The other (Pontecorvo, r950, r 952a, $b$, r955; Lewis, r951 ; Haldane, 1954) is that alleles between which crossing over occurs are mutants of functionally distinct chromosome segments controlling different steps of a reaction sequence which, owing to the nature of the reaction or reactants, can only take place by means of an " assembly line" process along the chromosome surface, and not between homologous chromosomes.

The widespread occurrence of the Lewis effect makes it important to determine which, if either, of these two alternatives is correct. One approach to the problem would be to obtain an estimate of the number of sites of mutation and crossing over within a single chromosome segment behaving as a functional unit. If this turned out to be very large the second alternative would become less likely.

In the present work a start along these lines was made with a number of adenine-requiring mutants of $A$. nidulans. Infertility of crosses involving allelic adenine-requiring mutants unfortunately prevented an adequate number of pairs of alleles being tested against each other for recombination for this purpose. On the other hand, information was obtained which suggests that multiple exchanges within very short chromosome segments may occur with a much greater frequency than expected (Pritchard, 1954). These observations may help in understanding of the mechanism of crossing over. 


\section{ANALYSIS OF MEIOTIC RECOMBINATION (i) Material and preliminary experiments}

Unless otherwise stated the techniques and notations are those used for the genetics of $A$. nidulans (Pontecorvo, 1953). In the present work two recessive mutants are termed allelic, if a heterozygote in trans or heterokaryon between them is mutant in phenotype. The present work is primarily concerned with nine allelic adenine-requiring mutants located very close to the locus "yellow" $(y)$ (fig. I) and obtained by Macdonald and Pontecorvo (1953) following U.V.-irradiation of a biotin-requiring strain $\left(b i_{1}\right)$. The mutants are designated as follows (isolation numbers in brackets) : $a d_{8}\left(\mathrm{~S}_{\mathrm{C}} 2\right) ; a d_{10}$ (S5D2) ; $a d_{11}$ (S5D3) ; $a d_{12}$ (S5C3) ; $a d_{16}$ (S5E5) ; $a d_{19}$ (S5E8); $a d_{20}$ (S6.2); $a d_{21}$ (S3Ar); $a d_{22}$ (S4A3). The first six were all derived from one irradiated suspension; they could have represented repeated isolations from a single mutant clone. The genetic analysis reported in this paper, however, indicates that at least five were mutants of independent origin. With the exception of $a d_{20}$, which was selected for study because it was phenotypically distinguishable from all but $a d_{16}$, the nine mutants are a random sample of the available adenine-requiring mutants located immediately to the right of $y$. Markers at other loci used in this work are indicated in fig. I. The map distances given were obtained by pooling data from a number of crosses (mainly unpublished data of other workers) giving homogeneous results.

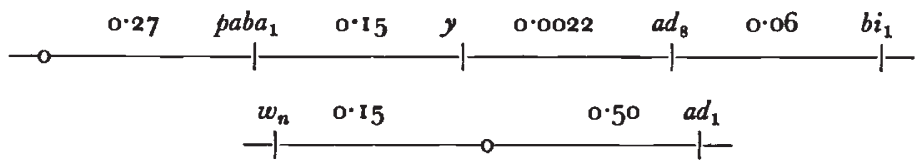

Fig. I.-Linkage relationships of certain loci of two chromosomes. o : centromere. $w_{n}$ (white) and $y$ (yellow) : conidium colour mutants (wild type green). $w_{n}$ epistatic to $y / Y$. $a d_{1}, a d_{8}, p a b a_{1}$, and $b i_{1}$ nutritional requirement mutants : $a d$, adenine ; $p a b a$, p-aminobenzoic acid (P.A.B.A.); bi, biotin. Two other markers not on the above chromosomes were also used : pyro, determining requirement for pyridoxin, and nic $_{2}$, determining requirement for nicotinic acid. The recombination fractions are in part based on work (unpublished) by other workers in the Department of Genetics, University of Glasgow. In the text, tables and subsequent figures the mutants $w_{n}, p a b a_{1}, b i_{1}, p y r o_{4}$, and $n i_{2}$, are referred to without their subscripts for convenience. Wild type alleles (all dominant) are indicated with capital letters.

Estimates of the recombination fractions between $y$ and each of the nine ad mutants were difficult to obtain owing to extremely close linkage and, with the exception of four, qualitative data only are available. Data concerning these four are given in table 1 .

The nine ad mutants cannot be distinguished by the qualitative test of response to alternative growth factors, growth being supported in all cases by adenosine, adenine or hypoxanthine, and to a limited extent by 4.amino-5. imidazole carboxamidine (kindly supplied by Dr Nimmo-Smith) which exerts a marked sparing effect on adenosine. Two mutants, $a d_{16}$ and $a d_{20}$, can grow to a limited extent on minimal medium (M.M.) on which they produce characteristic, slow-growing, aconidiate colonies and can therefore be distinguished from the rest. $a d_{20}$ is distinguishable from all the others since it is specifically suppressed by a recessive mutant on the other arm of the same chromosome and more than 50 map units away (Pritchard and Kafer, unpublished).

Three methods have been used to establish that the ad mutants are allelic. Firstly, balanced heterokaryons between strains carrying $a d_{8}$ and strains carrying each of the other ad mutants were synthesised on adenine-supplemented medium and then transferred to adenine-deficient medium. The heterokaryons were balanced on other nutritional requirements. In no case was good growth maintained on transfer to adenine-deficient medium although repeated tests were made. 
Secondly, diploids (Roper, 1952) heterozygous for three pairs of mutants ( $a d_{11}$ and $a d_{8} ; a d_{16}$ and $a d_{8} ;$ and $a d_{19}$ and $\left.a d_{8}\right)$ were synthesised; all had mutant phenotypes, i.e. indistinguishable from that due to the "higher" allele. Finally, from crosses between those pairs of mutants which were fertile (vide infra) no diploid adenine-independent types were obtained from platings of large numbers of ascospores on adenine-deficient medium. Many would have been expected if the

TABLE I

Recombination fractions between $\mathrm{y}$ and four alleles of the $\operatorname{ad}_{8}$ region

\begin{tabular}{|c|c|c|c|c|c|c|c|}
\hline \multirow{2}{*}{ Cross * } & \multirow{2}{*}{ Selection $\dagger$} & \multicolumn{5}{|c|}{ Segregations } & \multirow{2}{*}{$\begin{array}{l}\text { Recombination } \\
\text { fractions }\end{array}$} \\
\hline & & $y$ & $r$ & Total & $\begin{array}{l}\text { Cross- } \\
\text { overs }\end{array}$ & Total & \\
\hline 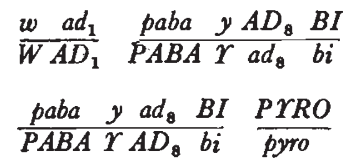 & $\begin{array}{c}W A D_{1} A D_{8} \\
A D_{8} P Y R O\end{array}$ & 1018 & $\begin{array}{r}2 \\
1681\end{array}$ & $\begin{array}{l}1020 \\
1685\end{array}$ & 6 & 2705 & $0.0022 \pm 0.00090$ \\
\hline 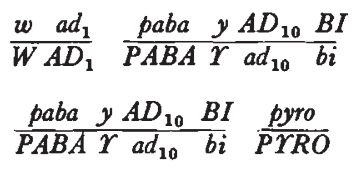 & $\begin{array}{l}W A D_{1} A D_{10} \\
A D_{10} P r R O\end{array}$ & $\begin{array}{r}471 \\
3455\end{array}$ & 10 & $\begin{array}{r}473 \\
3465\end{array}$ & 12 & $393^{8}$ & $0.003^{0} \pm 0.00088$ \\
\hline 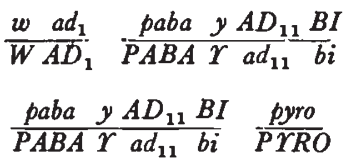 & $\begin{array}{l}W A D_{1} A D_{11} \\
A D_{11} P r R O\end{array}$ & 2305 & 9 & 2314 & 9 & 2368 & $0.003^{8} \pm 0.0013$ \\
\hline 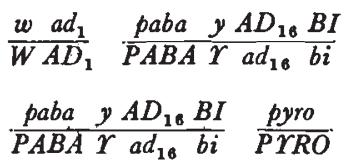 & $\begin{array}{l}W A D_{1} A D_{16} \\
A D_{16} P Y R O\end{array}$ & 1870 & $\mathbf{I}$ & 1871 & 1 & 2087 & $0.0004^{8} \pm 0.00048$ \\
\hline
\end{tabular}

* Symbols above the fraction signs give the genotype of one parent, those below that of the other.

$\dagger$ Ascospores from each cross were plated on minimal medium supplemented with p-aminobenzoic acid and biotin only.

All apparent recombinants between $y$ and ad were checked for diploidy. A few dipluids did arise in most crosses : they are not included in the table.

mutants had been physiologically non-allelic since in $A$. nidulans about $\mathrm{I}$ in every 100 ascospores is unreduced (Pritchard and Pontecorvo, 1953; Pritchard, I 953 and unpublished).

(ii) Crosses involving pairs of different ad alleles

Attempts to improve the fertility of crosses involving allelic ad mutants by variation of a number of conditions were all unsuccessful. A slight improvement in fertility was usually observed, however, when crossing was carried out on "sporulating minimal medium" (Pontecorvo, I953), and when the petri dishes containing the heterokaryons were partially sealed with "cellotape". All crosses between 
strains with different ad alleles were therefore carried out under these conditions.

$a d_{8}$ and $a d_{11}$. -The procedure adopted for crossing strains carrying these two mutants and the detection and isolation of adenineindependent types will be given as an example of the procedure adopted in subsequent crosses.

A balanced heterokaryon between two strains pabay $a d_{8}$ and $a d_{11} b i$ was obtained by inoculating conidia from both strains together on minimal medium supplemented with adenine only. The heterokaryon was transferred to petri dishes containing sporulating minimal medium plus adenine. 'The dishes were sealed with "cellotape" and incubated for at least three weeks at $37^{\circ}$.

Large numbers of perithecia were collected from the heterokaryon and washed in a I/I0oo solution of detergent ("calzolene oil ") to remove as many conidia as possible. The washed perithecia were crushed and the ascospores suspended in saline.

The suspension contained $2 \cdot 13 \times 10^{6} / \mathrm{ml}$. ascospores and approximately $7 \cdot 0 \times 10^{6} / \mathrm{ml}$. conidia. The heavy conidial contamination was unavoidable owing to the relative infertility of the cross which necessitated collecting many thousands of perithecia, each with very few ascospores but with many conidial heads adhering to it. Complete removal of adhering conidia by washing was not possible. $3.6 \mathrm{ml}$. of the suspension was added to molten agar minimal medium supplemented with p-aminobenzoic acid and biotin. This was poured as a top layer on ten petri dishes already containing $10 \mathrm{ml}$. solidified medium with the same supplements. Each dish was therefore inoculated with about $7.67 \times 10^{5}$ ascospores and $2.52 \times 10^{6}$ conidia.

From this plating (table 2) 365 adenine-independent colonies were obtained of which ro were green and 355 yellow. All the greens and a sample of $\mathbf{I} 79$ of the yellows were tested for nutritional requirements. One hundred and thirty-six of the tested yellows were crossovers between $y$ and $b i$ although the standard map distance between these loci is less than 6 units. There is clearly an association between the origin of adenine-independent colonies and crossing over between $y$ and $b i$. All of the crossovers between $y$ and $b i$ have the genotype $y b i$; there are no crossovers of the complementary type.

The simplest interpretation of these results is that $a d_{11}$ and $a d_{8}$ are mutants of different loci, the former located nearest to $y$, and that adenine-independent types can arise following an exchange between them. The absence of adenine-independent colonies with the genotype $r B I$ makes it unlikely that unequal crossing over is involved.

If the adenine-independent types arose exclusively as a result of crossing over between the alleles about 6 per cent. would be expected to have the parental association of markers $y B I$ in the absence of interference (i.e. double crossovers with one exchange between the alleles and a second between $a d_{8}$ and $b i$ ). In fact, over 24 per cent. of the yellows tested were of this type. Making the same assumptions, 
no adenine-independent colonies with $Y$ bi would be expected owing to the extremely close linkage between $y$ and $a d_{11}$, but io were obtained.

Back-mutants of $a d_{11}$ or $a d_{8}$ among the ascospores or conidia plated would also produce adenine-independent colonies with the

TABLE 2

Detection and estimation of recombinants from a cross involving $\mathrm{ad}_{11}$ and $\mathrm{ad}_{8}$

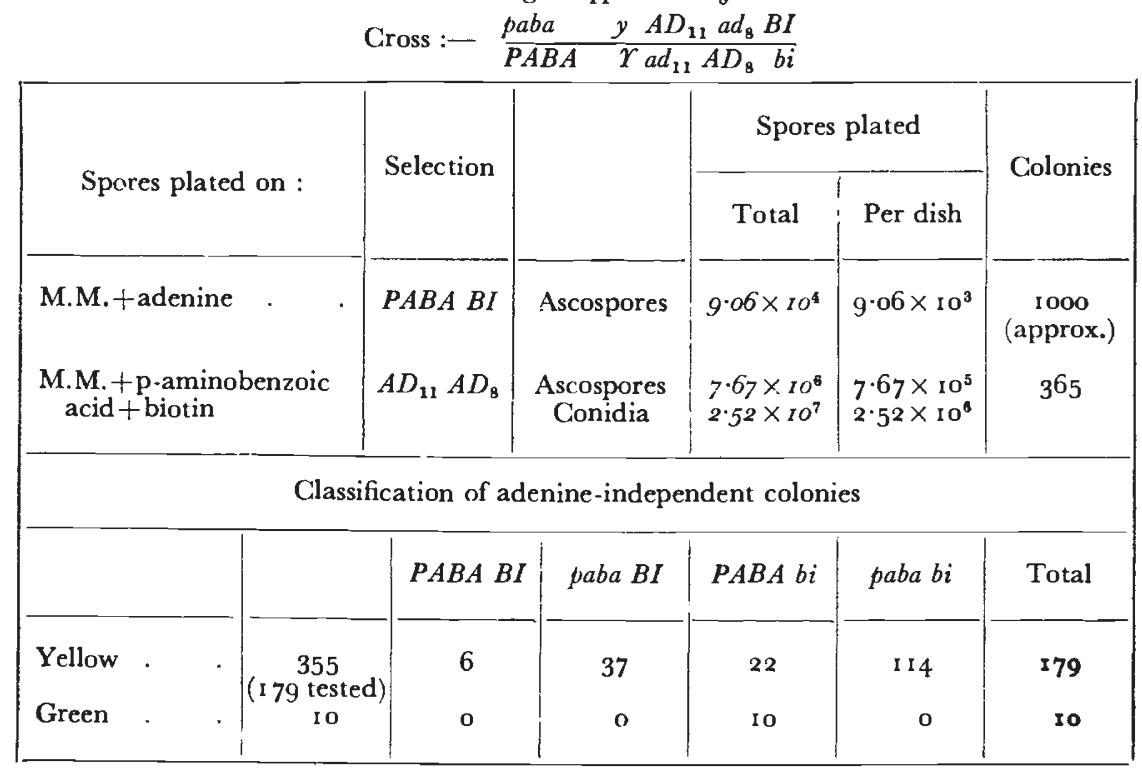

Estimation of the recombination fraction between $a d_{11}$ and $a d_{8}$ (see text p. 349) :

$\mathrm{n}=$ No. of ascospores plated on minimal med rum with adenine $=9.06 \times 10^{4}$

$a_{1}=$ No. of colonies produced by $n$ ascospores $\quad=$ rooo

$\mathrm{a}_{2}=\left(\mathrm{n}-\mathrm{a}_{1}\right)$

$\mathrm{m}=$ No. of ascospores plated on minimal medium with p-aminobenzoic acid and biotin $=7.67 \times 10^{8}$

$b_{1}=$ No. of colonies obtained from $m$ ascospores $=365$

$b_{2}=\left(m-b_{1}\right)$

$\mathbf{x}=$ Recombination fraction between paba and $b i . \quad A$ value of 0.2 has been used in this and subsequent calculations.

$\mathrm{h}=$ Fraction of ascospores viable and from hybrid asci $=2 \mathrm{a}_{\mathbf{1}} / \mathrm{nx}=0 \cdot \mathrm{II}$

S.E $E_{h}=\sqrt{\left(\mathrm{r} / \mathrm{a}_{1}+\mathrm{I} / \mathrm{a}_{2}\right) \cdot \mathrm{h}^{2}(2-\mathrm{hx})^{2} / 4}=\sqrt{\mathrm{h}(2-\mathrm{hx}) / \mathrm{nx}}=0 \cdot 0035$

$\mathrm{q}=$ Recombination fraction between $a d_{11}$ and $a d_{8}=\mathrm{nb}_{1} \mathrm{x} / \mathrm{ma}_{1}=0 \cdot 00086$

$\mathrm{S} . \mathrm{E}^{2}=\sqrt{\mathrm{q}[\mathrm{nx}(2-\mathrm{hq})+\mathrm{mq}(2-\mathrm{hx})] / \mathrm{mnhx}}=0 \cdot 000053$

parental combinations $Y b i$ and $y B I$ and might thus account for the excess of these types obtained. Platings of large numbers of conidia from both parental strains (table 3), however, and also from the heterokaryon from which the ascospores had been obtained (table 3) failed to yield any adenine-independent colonies. The plating from the heterokaryon was made in view of the possibility that backmutation during its growth could have produced a clone of nonmutant nuclei.

Clearly the excess of adenine-independent colonies with the parental combinations of markers $Y b i$ and $y B I$ could scarcely have 
been due to back-mutation unless the mutation frequency were enormously greater in ascospores than conidia. Thus, even if the viability of ascospores was Ioo per cent. (certainly an overestimate),

TABLE 3

Summary of available data regarding back-mutation of a number of alleles of $a_{8}$

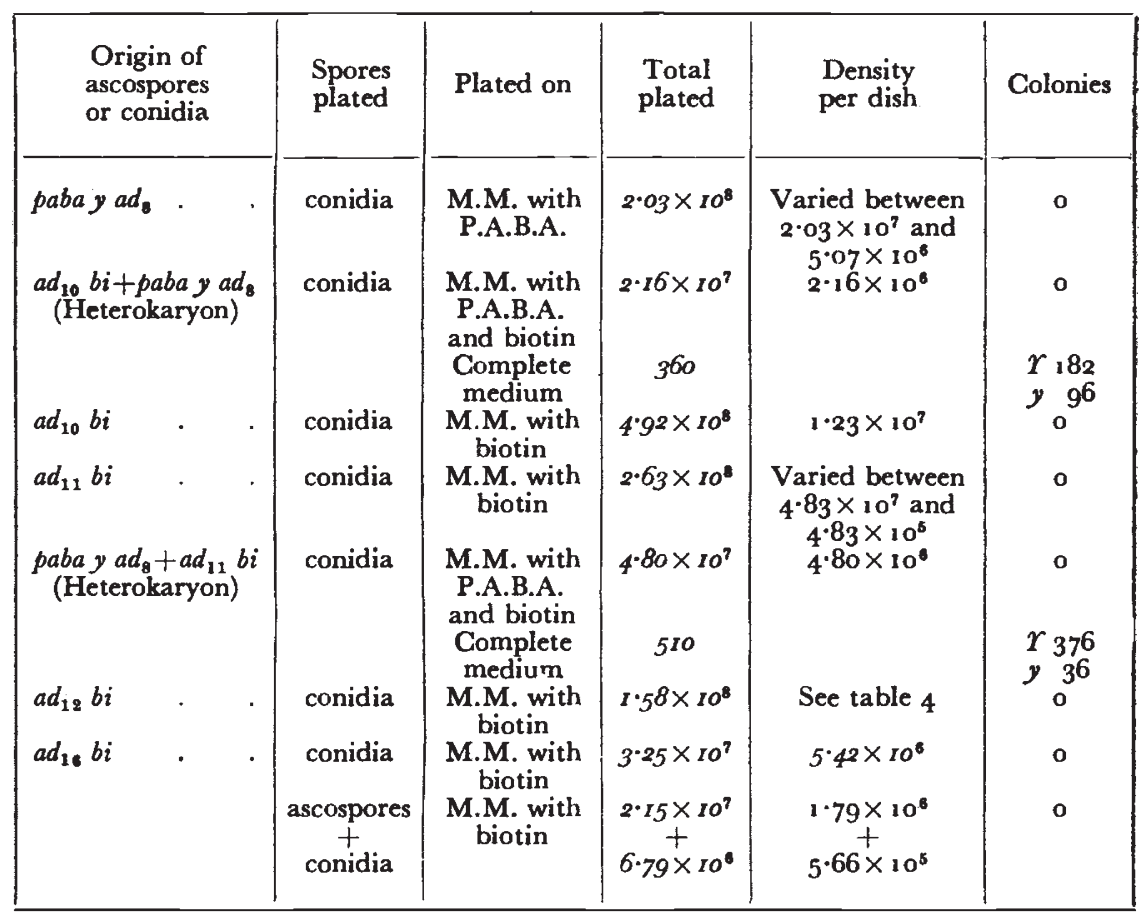

and if 9 of the $43 y B I$ colonies are considered to be double crossovers, the frequency of back-mutation of $a d_{8}$ among ascospores would be nearly $10^{-4}$. It is possible, however, that rare back-mutant nuclei in the heterokaryon would have a greater than random chance of participating in perithecium production and that the perithecia so produced would give rise to a greater number of ascospores than those originating from two ad nuclei.

That adenine-independence was not due to mutation at a nonlinked or loosely-linked suppressor locus, except perhaps in a small proportion of colonies, was shown by outcrossing to wild type a number of adenine-independent colonies of each class with respect to their $Y / y$ and $B I / b i$ genotype. No adenine-requiring segregants were obtained from any of these crosses.

That colonies with the phenotypes $Y$ or $B I$ were not heterozygous diploids was shown by measuring the conidium diameter of all such types. None was outside the haploid range (Pontecorvo et al., I954).

Further discussion on the origin of the large number of adenineindependent colonies carrying the parental combinations of markers 
will be deferred until the results obtained from similar crosses involving other pairs of alleles have been considered.

The recombination fraction between $a d_{11}$ and $a d_{8}$ was estimated in the following manner (Roper, 1953). It was assumed in the first instance that all adenine-independent colonies were recombinants between the two alleles.

A dilution of the original suspension of ascospores was spread in $0.1 \mathrm{ml}$. amounts over the surface of Io petri dishes containing solid minimal medium supplemented with adenine, and the number of colonies produced was counted. These are recombinants $P A B A B I$. Taking the recombination fraction between $p a b a$ and $b i$ as 0.2 , the

TABLE 4

Experiment to test for inhibition of an adenine-independent strain by conidia of an adenine-requiring strain

\begin{tabular}{|c|c|c|c|}
\hline $\begin{array}{l}\text { Conidia } \\
\text { plated on : }\end{array}$ & $\begin{array}{c}\text { Estimated conidia } \\
\text { per dish from } \\
a d_{12} b i\end{array}$ & $\begin{array}{c}\text { Estimated conidia } \\
\text { per dish from } \\
y b i\end{array}$ & $\begin{array}{c}\text { Colonies } \\
\text { (all yellow) }\end{array}$ \\
\hline M.M. + biotin & $\begin{array}{c}9.50 \times 10^{4} \\
4.75 \times 10^{8} \\
9.50 \times 10^{5} \\
4.75 \times 10^{8} \\
9.50 \times 10^{6} \\
4.75 \times 10^{2} \\
9.50 \times 10^{7} \\
\text { (two dishes) }\end{array}$ & 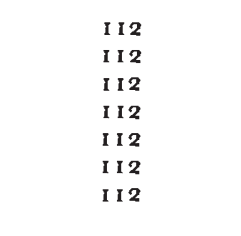 & $\begin{array}{l}\text { I I } 7 \\
\text { I } 23 \\
\text { I } 45 \\
\text { I } 29 \\
\text { I } 24 \\
\text { I } 41 \\
237\end{array}$ \\
\hline
\end{tabular}

number of colonies counted will represent one-half of this fraction, i.e. $\mathrm{O} \cdot \mathrm{I}$ of the viable ascospores plated of crossed origin. The proportion of ascospores of this type in the original suspension can therefore be estimated, and the recombination fraction between the two alleles calculated in the manner shown in table 2. I am indebted to $\mathrm{Dr}$ A. Durrant for the method of calculating the standard error of this estimate.

Calculation of the recombination fraction in this way is open to a number of sources of error such as inaccuracy of dilution and plating, differential viability of ascospores of different genotypes on different media, and at different density of plating ( $c f$. Grigg, I952), and probably most serious, variation of the recombination fraction between $p a b a$ and $b i$ from the standard value. In the present work, however, qualitative rather than quantitative information was required. The experimental data in table 4 indicate that inhibition of adenineindependent strains by high concentrations of adenine-requiring conidia ("Grigg effect") does not occur at concentrations used in this work.

$a d_{8}$ and $a d_{12}, a d_{16}, a d_{19}, a d_{20}$. -These four crosses were carried out in exactly the manner previously described, the strain paba $y a d_{8}$ being crossed to $a d_{12} b i, a d_{16} b i, a d_{19} b i$ and $a d_{20} b i$. The data are given in table 5 . 
TABLE 5

Detection and estimation of recombination in crosses involving $\mathrm{ad}_{8}$ and each of four other alleles

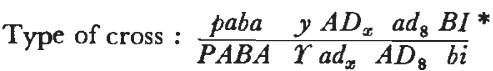

\begin{tabular}{|c|c|c|c|c|c|c|c|}
\hline \multirow{2}{*}{$\begin{array}{c}\text { Allele } \\
\text { tested } \\
\text { against } \\
\operatorname{ad}_{8}\end{array}$} & & \multicolumn{2}{|c|}{$\begin{array}{l}\text { Spores on M.M. }+ \\
\text { p-aminobenzoic acid } \\
+ \text { biotin (selecting } \\
\left.\qquad D_{x} A D_{\mathrm{g}}\right)\end{array}$} & \multirow[t]{2}{*}{ Colonies } & \multirow{2}{*}{$\begin{array}{c}\text { Ascospores } \\
\text { on M.M. }+ \\
\text { adenine } \\
\text { (selecting } \\
P A B A B I \text { ) }\end{array}$} & \multirow[t]{2}{*}{ Colonies } & \multirow{2}{*}{$\begin{array}{l}\text { Recom- } \\
\text { bination } \\
\text { fraction }\end{array}$} \\
\hline & & Total & Per dish & & & & \\
\hline$a d_{12}$ & $\begin{array}{l}\text { Ascospores } \\
\text { Conidia } \\
\text { Ascospores } \\
\text { Conidia } \\
\text { Total } \\
\text { Ascospores } \\
\text { Conidia }\end{array}$ & $\begin{array}{l}3 \cdot 60 \times 10^{6} \\
1 \cdot 44 \times 10^{7} \\
6 \cdot 00 \times 10^{5} \\
2 \cdot 40 \times 10^{6} \\
4.20 \times 10^{6} \\
1 \cdot 68 \times 10^{7}\end{array}$ & $\begin{array}{l}4.00 \times 10^{5} \\
1.60 \times 10^{6} \\
2 \cdot 00 \times 10^{5} \\
8.00 \times 10^{5}\end{array}$ & $\begin{array}{l}\text { I I I } \\
28\end{array}$ & $5.11 \times 10^{4}$ & 282 & $\begin{array}{c}0.0012 \pm \\
0.00012\end{array}$ \\
\hline $\operatorname{ad}_{1 \mathrm{~B}}$ & $\begin{array}{l}\text { Ascospores } \\
\text { Conidia } \\
\text { Ascospores } \\
\text { Conidia } \\
\text { Ascospores } \\
\text { Conidia } \\
\text { Total } \\
\text { Ascospores } \\
\text { Conidia }\end{array}$ & $\begin{array}{l}6 \cdot 34 \times 10^{6} \\
1 \cdot 15 \times 10^{6} \\
3 \cdot 17 \times 10^{6} \\
5.75 \times 10^{5} \\
3 \cdot 17 \times 10^{6} \\
5.75 \times 10^{5} \\
\\
1 \cdot 27 \times 10^{7} \\
2 \cdot 30 \times 10^{6}\end{array}$ & $\begin{array}{l}1.58 \times 10^{6} \\
2.87 \times 10^{6} \\
7.92 \times 10^{5} \\
1.44 \times 10^{5} \\
3.96 \times 10^{5} \\
7 \cdot 19 \times 10^{4}\end{array}$ & $\begin{array}{r}65 \\
3^{6} \\
3^{8} \\
139\end{array}$ & $3.34 \times 10^{4}$ & $5^{2}$ & $\begin{array}{c}0.0014 \pm \\
0.00042\end{array}$ \\
\hline$a d_{19}$ & $\begin{array}{l}\text { Ascospores } \\
\text { Conidia }\end{array}$ & $\begin{array}{l}4.20 \times 10^{6} \\
I \cdot 20 \times 10^{7}\end{array}$ & $\begin{array}{l}8.40 \times 10^{5} \\
2 \cdot 40 \times 10^{6}\end{array}$ & 28 & $5 \cdot 20 \times 10^{4}$ & $5^{6}$ & $\begin{array}{c}0.0012 \pm \\
0.00029\end{array}$ \\
\hline$a d_{20}$ & $\begin{array}{l}\text { Ascospores } \\
\text { Conidia }\end{array}$ & $\begin{array}{l}3.94 \times 10^{6} \\
I \cdot 22 \times 10^{6}\end{array}$ & $\begin{array}{l}6.56 \times 10^{5} \\
2.03 \times 10^{5}\end{array}$ & $80 \dagger$ & $9.37 \times 10^{4}$ & $23^{6}$ & $\begin{array}{c}0 \cdot 0016 \pm \\
0 \cdot 00021\end{array}$ \\
\hline \multicolumn{8}{|c|}{ Classification of adenine-independent colonies } \\
\hline $\begin{array}{c}\text { Allele } \\
\text { tested } \\
\text { against } \\
\text { ad }_{8}\end{array}$ & & $P A B A B I$ & $p a b a B I$ & $P A B A b i$ & haba bi & \multicolumn{2}{|c|}{ Totals } \\
\hline$a d_{12}$ & $\begin{array}{l}\text { yellow } \\
\text { green }\end{array}$ & $\begin{array}{l}7 \\
1\end{array}$ & $\begin{array}{r}24 \\
0\end{array}$ & $\begin{array}{r}17 \\
4\end{array}$ & $\begin{array}{r}85 \\
1\end{array}$ & $\left.\begin{array}{r}133 \\
6\end{array}\right\}$ & 139 \\
\hline$a d_{16}$ & $\begin{array}{l}\text { yellow } \\
\text { green }\end{array}$ & $\begin{array}{l}7 \\
0\end{array}$ & $\begin{array}{r}24 \\
0\end{array}$ & $\begin{array}{r}16 \\
4\end{array}$ & $\begin{array}{r}86 \\
2\end{array}$ & $\begin{array}{r}133 \\
6\end{array}$ & 139 \\
\hline$a d_{18}$ & $\begin{array}{l}\text { yellow } \\
\text { green }\end{array}$ & $\begin{array}{l}2 \\
0\end{array}$ & $\begin{array}{l}8 \\
0\end{array}$ & $\begin{array}{l}4 \\
1\end{array}$ & $\begin{array}{r}13 \\
0\end{array}$ & $\begin{array}{l}27) \\
1\}\end{array}$ & 28 \\
\hline$a d_{20}$ & $\begin{array}{l}\text { yellow } \\
\text { green }\end{array}$ & $\begin{array}{l}2 \\
0\end{array}$ & $\begin{array}{r}10 \\
1\end{array}$ & $\begin{array}{r}24 \\
6\end{array}$ & $\begin{array}{r}35 \\
\mathbf{I}\end{array}$ & $\left.\begin{array}{r}7 I \\
8\end{array}\right\}$ & 79 \\
\hline
\end{tabular}

* $a d_{x}$ represents either $a d_{12}, a d_{16}, a d_{19}$ or $a d_{20}$.

$\dagger$ One colony $(Y \quad r i)$ had a growth rate less than wild type and was stimulated by adenine. It probably carried a suppressor. It is not included in the lower table or in the estimate of the recombination fraction. 
Adenine-independent colonies arose in each cross. In each case there was a clear association between adenine-independence and crossing over between $y$ and $b i$, the simplest interpretation of the data being that $a d_{8}$ lies to the right of each of the other four alleles.

It is remarkable that the relative frequency of the four possible genotypes with respect to $y$ and $b i$ among the adenine-independent colonies is very similar in each cross (embarrassingly so in the case of the crosses to $a d_{12}$ and $a d_{16}$ ). The data from all five crosses so far discussed involving $a d_{8}$ are in fact statistically homogeneous in this respect; the high proportion of colonies carrying the parental combinations of the markers $y$ and $b i$ being consistently obtained.

In view of the fact found later that reversion of $a d_{20}$ strains due to suppressor mutation occurs frequently, it cannot be excluded that a proportion of the $Y$ bi colonies obtained from the cross involving this mutant were of this type. In fact, one had a sub-normal growth rate and was stimulated by adenine. Two others, outcrossed to an adenine-independent strain, gave no ad progeny.

$a d_{8}$ and $a d_{10}$. - The proportion of adenine-independent colonies from this cross was extremely low (less than ${ }^{10^{-5}}$ ) and the data in table 6 are the results of four separate platings of ascospores.

Out of 28 adenine-independent colonies obtained I4 were $Y B I$. This suggests that $a d_{10}$ lies at a locus to the right of $a d_{8}$ representing a third locus in the series. However, the occurrence of 3 colonies with the complementary genotype $(y b i)$ suggests that unequal crossing over may be involved in this case.

A high proportion of colonies with the parental combinations $y B I$ and $Y b i$ was again encountered. The massive numbers of ascospores and conidia involved in platings from this cross increased the possibility that back-mutants might be contributing to these classes, but platings of conidia from both parental strains and from the heterokaryon from which the ascospore suspension was obtained (table 3 ) indicated a very low back-mutation rate in conidia.

$a d_{11}$ and $a d_{16}$. -With the exception of the cross involving $a d_{16}$ and $a d_{11}$, attempts to cross together in pairs the four alleles located to the left of $a d_{8}$ failed due to infertility.

The technique in the cross involving $a d_{16}$ and $a d_{11}$ was slightly different from that previously used. The presence of an additional marker pyro, made possible a more reliable estimate of the percentage of viable ascospores from hybrid asci (table 7). In addition, exclusion of pyridoxin from the medium when selecting for adenine-independent types cut down the background growth caused by conidia from the $a d_{16}$ parent and by ascospores from zygotes of selfed $a d_{16}$ origin.

Adenine-independence was again associated with crossing over between $y$ and $b i$, the data indicating that $a d_{16}$ is located to the left of $a d_{11}$. The proportion of colonies with the parental combinations $y B I$ and $Y b i$ was again high although a low back-mutation rate for $a d_{18}$ both in ascospores and conidia is indicated by the data in table 3 . 


\section{(iii) Discussion}

In each of the seven crosses so far analysed the origin of adenineindependent colonies was associated with recombination between $y$

TABLE 6

Detection and estimation of recombination in a cross involving $\mathrm{ad}_{8}$ and $\mathrm{ad}_{10}$

Cross : \begin{tabular}{lll}
$p a b a$ & $y a d_{8} A D_{10}$ & $B I$ \\
\hline$P A B A$ & $Y A D_{8} a d_{10}$ & $b i$
\end{tabular}

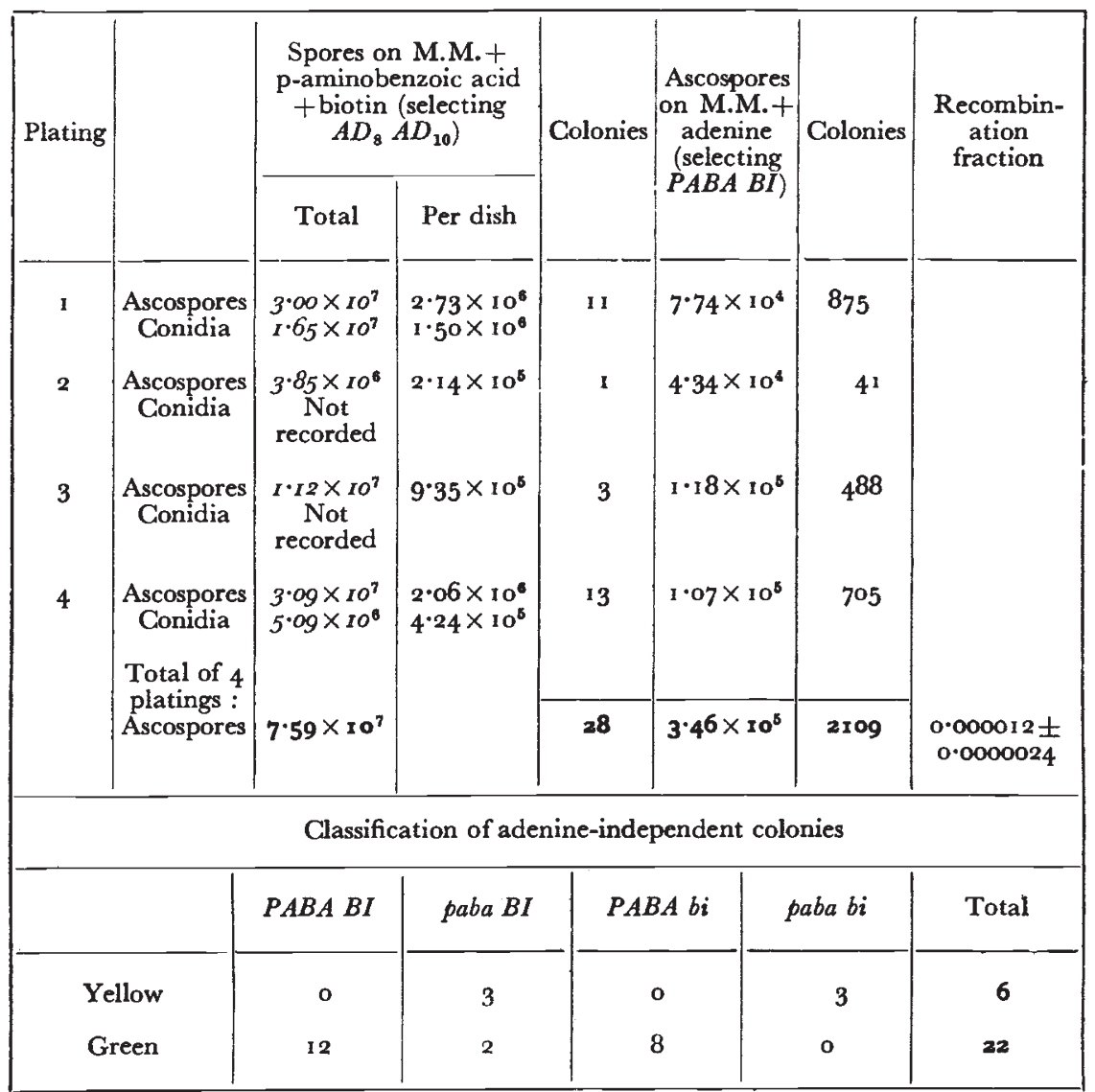

and $b i$. Nevertheless the proportion of such colonies carrying the parental combinations $y B I$ and $Y b i$ was in every case much higher than expected on the assumptions that all adenine-independent types resulted from crossing over between alleles and that there was no interference. The first suggestion which comes to mind is that a proportion of the adenine-independent colonies originated by backmutation. The following evidence, however, makes this interpretation unlikely.

First, the data summarised in table 3 indicate a very low backmutation rate among conidia for all alleles so far tested. In one case, 
$a d_{16}$ (table 3 ), the incidence of back-mutation was also tested among ascospores: no back-mutant was present in $2 \cdot 15 \times 10^{7}$ ascospores. (It was not possible to make this test with other alleles owing to selfinfertility.)

Second, the relative frequencies of the four genotypes $y b i, r B I$,

TABLE 7

Detection and estimation of recombination in a cross involving $\mathrm{ad}_{16}$ and $\mathrm{ad}_{11}$

Cross : \begin{tabular}{llll}
$p a b a$ & $y A D_{16} a d_{11}$ & $B I$ & $P Y R O$ \\
\hline$P A B A$ & $Y_{16} a d_{16} A D_{11}$ & $b i$ & $\frac{P y r o}{p y}$
\end{tabular}

\begin{tabular}{|c|c|c|c|c|c|c|}
\hline \multirow{2}{*}{$\begin{array}{l}\text { Spores } \\
\text { plated on }\end{array}$} & \multirow{2}{*}{ Selection } & & \multicolumn{2}{|c|}{ Spores plated } & \multirow{2}{*}{ Colonies } & \multirow{2}{*}{$\begin{array}{l}\text { Rec. } \\
\text { fract.* }\end{array}$} \\
\hline & & & Total & Per dish & & \\
\hline $\begin{array}{l}\text { M.M. + p- } \\
\text { aminobenzoic } \\
\text { acid + biotin }\end{array}$ & $A D_{16} A D_{11}$ PYRO & $\begin{array}{c}\text { Ascospores } \\
\text { Conidia }\end{array}$ & $\begin{array}{l}3.71 \times 10^{7} \\
1.57 \times 10^{7}\end{array}$ & $\begin{array}{l}3.09 \times 10^{6} \\
1.31 \times 10^{6}\end{array}$ & 57 & $\begin{array}{c}0.00089 \\
\pm \\
0.00028\end{array}$ \\
\hline $\begin{array}{l}\text { M.M. + aden- } \\
\text { ine + biotin }\end{array}$ & PABA PYRO & Ascospores & $8 \cdot 19 \times 10^{4}$ & $1.36 \times 10^{4}$ & $14^{1}$ & \\
\hline \multicolumn{7}{|c|}{ Classification of adenine-independent colonies } \\
\hline & $P A B A B I$ & paba $B I$ & $P A B A b i$ & paba $b$ & & Total \\
\hline Yellow & $\mathbf{I}$ & 5 & 4 & $3^{6}$ & & 46 \\
\hline Green & 0 & o & 10 & I & & II \\
\hline
\end{tabular}

* In this cross the fraction of viable ascospores from hybrid asci is $4 a_{1} / \mathrm{n}$; the recombin-

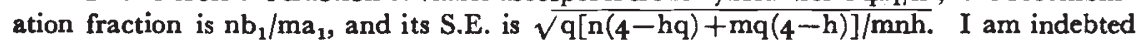
to Dr O. Frydenberg for the calculation of this standard error. Symbols as in table 2.

$y B I$ and $Y b i$ among adenine-independent colonies from the five crosses involving $a d_{8}$ and the alleles to the left (tables 2 and 5) are statistically homogeneous. This would not be expected if backmutants contributed appreciably to the two parental classes $y B I$ and $Y b i$ since back-mutant nuclei would have a clonal distribution and their frequency would be subject to considerable fluctuation in different crosses (Luria and Delbruck, 1943).

Finally, and most significant, is a comparison of the data from the crosses between $a d_{8}$ and the alleles to the left on the one hand, and between $a d_{8}$ and $a d_{10}$ on the other. The frequency of adenineindependent colonies obtained from the former crosses is greater by a factor of nearly $1 \mathrm{O}^{2}$ than in the latter. If the majority of $y B I$ colonies obtained in the former was due to back-mutation of $a d_{8}$, then in the latter cross the proportion of such types to crossover types should be very much higher. This was not found, however; the proportion 
of the two types remains approximately the same. Such a result is inconsistent with the view that the majority of $y B I$ colonies are backmutants of $a d_{8}$.

Four other alternatives which might account for the observed results were therefore considered; unequal sister strand crossing over ; the presence of rearrangements ; negative interference ; and some form of gene conversion or transformation.

Unequal sister strand crossing over might be capable of producing adenine-independent colonies with the parental combinations of the markers $y$ and $b i$, but the argument against back-mutation applies equally well here, viz. that the frequency of such an event should be independent of the frequency of recombination between the alleles.

An inversion in one or both strains used to test two alleles against each other might account for the large proportion of adenineindependent colonies not apparently associated with crossing over between $y$ and $b i$ by elimination of a proportion of the single crossovers between the alleles. It has proved impossible to construct a model for any simple system of rearrangements that will satisfactorily account for the results obtained from crosses involving pairs of alleles, taking into account that crosses involving each ad mutant and an adenine-independent strain gave no evidence of the presence of inversions. More conclusive evidence against this alternative was obtained from the results of mitotic analysis (see section 3 ).

Negative interference would need to be very intense to account for the observed results. Thus the greatest observed value for the recombination fraction between $y$ and any ad mutant was 0.0038 (table I). The smallest value for the same recombination fraction computed among crossovers between two ad mutants was 0.027 (from the cross involving $a d_{11}$ and $a d_{8}$ in which there were Io $Y b i$ colonies among 365 adenine-independent). It should be realised that the term "negative interference" is merely a description, not an explanation, of the fact that a crossover betwen two ad alleles is associated with one or more further exchanges nearby more often than by chance.

A distinction between negative interference, mutation and gene transformation (or in other words, a high mutation rate in heterozygotes) could be made by means of tetrad analysis, but the low incidence of adenine-independence in crosses involving alleles and the infertility of these crosses makes this impracticable. On the other hand, the occurrence of mitotic crossing over in heterozygous diploids of $A$. nidulans makes the analysis of half-tetrads possible and at the same time allows the automatic selection of half-tetrads in which a change of phenotype from adenine-requirement to adenineindependence has occurred (Roper and Pritchard, I955). An analysis of this type was therefore undertaken. It was based on the assumption that crossing over is substantially the same process at meiosis and at mitosis. 


\section{ANALYSIS OF MITOTIC RECOMBINATION}

\section{(i) Introduction}

Following the synthesis of heterozygous diploid strains of $A$. nidulans (Roper, 1952) and the discovery of mitotic segregation in these (Pontecorvo and Roper, I953), the analysis of the mechanisms of mitotic segregation has now progressed to the point where this process can be used for genetic analysis outside the sexual cycle (Pontecorvo, Roper and Forbes, 1953; Pontecorvo, Tarr Gloor and Forbes, 1954; Pontecorvo and Kafer, 1954).

The purpose of the experiments describe 1 in this section was threefold. Firstly, to test the possibility that " negative interference" was involved in the origin of adenine-independent colonies not associated with recombination in crosses involving pairs of alleles. Secondly, to explore the possibility of using mitotic crossing over as a method of genetic analysis of the linear relationships between alleles of $a d_{8}$, thereby circumventing the obstacle of infertility. Finally, to obtain recombinants carrying two ad alleles in coupling, thereby making it possible to follow the segregation of three alleles simultaneously. They would also make it possible to verify what had been assumed from the fact that all the ad alleles are recessive, namely that a strain heterozygous for two alleles in cis would be adenineindependent.

\section{(ii) Techniques}

In the following experiments three operations were involved. The first was to obtain a diploid heterozygous for two ad alleles. The second was to obtain adenine-independent diploid colonies from such a diploid. The third was to determine the genotype of these colonies.

Two diploids heterozygous for $a d_{8}$ and $a d_{16}$ were synthesised (see Roper, 1952). These two alleles were chosen because they are phenotypically distinguishable which, as will become apparent, was a decisive advantage. The diploids had the following genotypes :

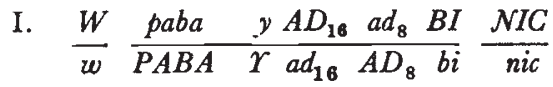

$$
\begin{aligned}
& \text { II. } \frac{p a b a}{P A B A} \Upsilon^{Y a d_{16} A D_{8} \quad b i} \frac{\text { y } A d_{8} B I}{\text { pyro }}
\end{aligned}
$$

As expected they were adenine-requiring and on minimal medium were intermediate in phenotype between $a d_{8}$ and $a d_{16}$.

When selecting adenine-independent colonies from these diploids doubly heterozygous in trans, it was important to avoid isolating from a single clone more than once. To this end, conidia from either diploid I or II were plated, about ro per dish, on agar medium lacking adenine. The conidia gave rise to slow growing aconidiate colonies, and when these were about $\mathrm{x} \mathrm{cm}$. in diameter they were covered by a thin layer of the same medium. During further incubation very slow growth of the colonies occurred through the covering layer of medium. Hyphæ in which "adenine-independent nuclei" were present would have an enormous selective advantage over hyphæ with only "adenine-dependent nuclei" and would reach the surface first, giving rise to rapidly growing conidiate colonies. Such reversions were readily obtained and a single-conidium isolation made from each. Since only one adenine-independent strain was isolated from a single adenine-requiring colony, repeated isolation from a single clone was excluded. Only about ro per cent. of 
the colonies produced adenine-independent recombinants by this technique, indicating that the rate with which these arise is very low.

The techniques employed for the determination of the genotype of the adenineindependent diploids obtained from the diploids I and II were those in routine use in $A$. nidulans (Pontecorvo et al., 1954; Pontecorvo and Kafer, 1954) and will not be redescribed here. The determination of the genotype with respect to $a d_{s}$ and $a d_{16}$ requires explanation, however.

As is made clear in fig. 2, adenine-independent diploids of two types would be expected, presumably with equal frequency, following a single mitotic exchange between the two alleles. One carries the reciprocal products of a mitotic exchange between the two alleles, the other has one crossover and one non-crossover strand. A number of diploids apparently of the former type were obtained, i.e. they were prototrophs (a diploid of the latter type would be biotin-requiring), they had the

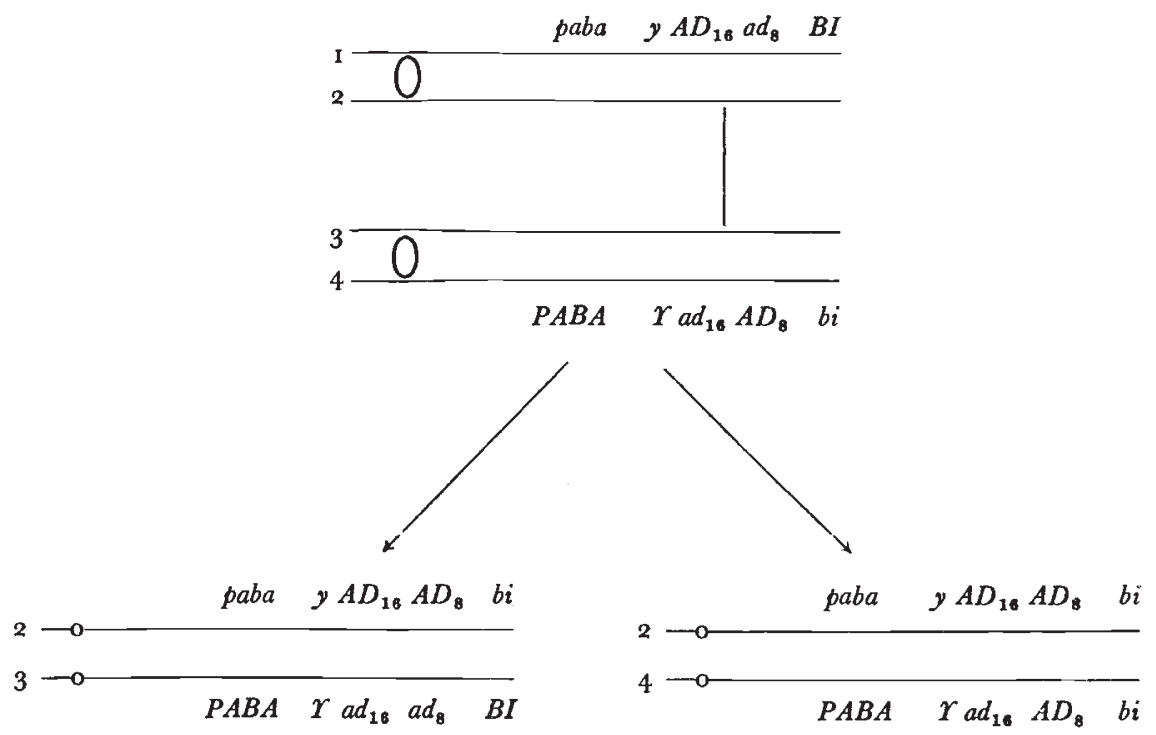

Fig. 2.-Types of diploid segregants produced following a mitotic exchange between $a d_{16}$ and $a d_{8}$. In this and subsequent figures the parent diploid is shown with the two homologous chromosomes divided into chromatids joined at the centromere. The four chromatids are numbered. In the segregant diploids the two homologues are shown as single strands. Segregants not possessing chromatid 2 are not shown as they will be adenine-requiring.

constitution $y b i / Y B I$ and therefore carried the complementary products of a mitotic exchange between $y$ and $b i$, and the $y b i$ strand carried the wild type alleles of $a d_{16}$ and $a d_{8}$, suggesting that the mitotic exchange had taken place between the two alleles. Such diploids should carry the two alleles $a d_{16}$ and $a d_{8}$ in $c i$ on the other strand, i.e. that carrying $Y$ and $B I$. Haploids carrying the $Y B I$ strand from all diploids of this type were adenine-requiring and the majority had a phenotype indistinguishable from that of $a d_{8}$. In the parent diploid $a d_{8}$ was in coupling with $y$ but it was now apparently in coupling with $r$. This was again suggestive that the $Y B I$ strand in segregant diploids of this type carried both $a d_{16}$ and $a d_{8}$ and, as might be expected, that the phenotype of haploids carrying both these alleles in cis was identical with that of the more extreme of the two alleles $\left(a_{8}\right)$. Proof of this was obtained by outcrossing a haploid of this type to an adenine-independent strain and recovering the less extreme mutant $\left(a d_{16}\right)$.

The technique for separating $a d_{16}$ from $a d_{8}$ was as follows. Since recombination between the two alleles is rare, the technique had to be selective. From a suspected 
double mutant with the genotype $\left(a d_{16}\right) a d_{8}$ pyro, a recombinant $\left(a d_{16}^{\prime}\right) a d_{8} b i$ was obtained. This was outcrossed to a strain paba $y$. Ascospores were plated on minimal medium supplemented with adenine and any green colonies which arose were tested for adenine-requirement (table 8). These colonies $(\Upsilon B I)$ are all crossovers between $y$ and $b i$. Since the ratio of the distances between $a d_{16}$ and $a d_{8}$ on the one hand and $a d_{8}$ and $b i$ on the other is about $1: 50$, about 2 per cent. of the colonies tested should be crossovers between $a d_{16}$ and $a d_{8}$ and carry the single mutant $a d_{16}$ if it is present. In fact $3 a d_{16} A D_{8}$ recombinants were obtained out of 129 colonies tested. The presence of $a d_{16}$ in the double mutant was therefore proved.

The result shows beyond doubt that a heterozygote with $a d_{16}$ and $a d_{8}$ in $c i$ is wild type while the heterozygote in trans is mutant, and the Lewis effect is therefore established in this case.

TABLE 8

Recovery of $\operatorname{ad}_{16}$ from a double mutant $\operatorname{ad}_{16} \operatorname{ad}_{8}$

Cross : \begin{tabular}{llll}
$P A B A$ & $Y a d_{18}$ & $a d_{8}$ & $b i$ \\
\hline$p a b a$ & $y A \bar{D}_{16} A D_{8}$ & $B I$
\end{tabular}

\begin{tabular}{|c|c|c|c|c|c|}
\hline \multirow{2}{*}{$\begin{array}{c}\text { Ascospores } \\
\text { plated on: }\end{array}$} & Selection & \multicolumn{3}{|c|}{ Segregation } & \multirow{2}{*}{ Total } \\
\cline { 2 - 5 } & $A D_{16} A D_{\mathrm{d}}^{*}$ & $a d_{16} A D_{8}$ & $\begin{array}{c}A D_{16} a d_{8} \\
\text { or } a d_{18} a d_{8}\end{array}$ & \\
\hline M.M. +adenine & $P A B A Y B I$ & 1 & 3 & 125 & 129 \\
\hline
\end{tabular}

* Diploids (6) with this phenotype were also obtained.

(iii) Results

A total of 43 adenine-independent diploids was obtained in the manner already described from one or other of the parent diploids. The genotype of all but two of these was determined by further analysis. They fell into 13 classes with respect to their phenotype and genotype (table 9) and the origin of each class will be considered in turn. In what follows the chromosome of a diploid carrying $y$ will be called the yellow strand and that carrying $r$, the green strand.

CLASS I.-The origin of diploids of this type has already been discussed. They carry the reciprocal products of a mitotic exchange between the alleles. The genotype $a d_{16} a d_{8}$ has been inferred from the fact that a haploid carrying the green strand, or a diploid homozygous for this strand, has a phenotype identical with that of $a d_{8}$, since this allele was in coupling with $y$ in the parent diploid. In one case the presence of $a d_{16}$ has been proved by outcrossing as described before (table 8).

ClaAss II.-Diploids of this type have the genotype expected following a single mitotic exchange between the alleles and inclusion of one crossover and one non-crossover product in the same daughter nucleus (see fig. 2).

It should be noted that whereas 16 diploids of Class ii were obtained, there were only 8 in Class i. On the assumption that normal mitotic segregation of the centromeres occurs after a mitotic exchange, 
equal numbers of these types were expected. Although the deviation is not significant, it is possible that the two chromatids involved in one mitotic crossover tend to segregate to opposite poles with greater than random frequency.

TABLE 9

The phenotype and genotype of adenine-independent diploids obtained from the two parent diploids heterozygous for $\operatorname{ad}_{16}$ and $\operatorname{ad}_{8}$

\begin{tabular}{|c|c|c|c|}
\hline Class no. & Phenotype & Genotype * & No. obtained \\
\hline $\mathrm{i}$ & wild type & $\begin{array}{lllll}p a b a & y & A D_{16} & A D_{\mathrm{B}} & b i \\
P A B A & Y & a d_{16} & a d_{\mathrm{B}} & B I\end{array}$ & 8 \\
\hline ii & biotin-requiring & $\begin{array}{llll}p a b a & y A D_{16} A D_{\mathrm{B}} & b i \\
P A B A & Y a d_{16} A D_{8} & b i\end{array}$ & 16 \\
\hline iii & wild type & $\begin{array}{lllll}p a b a & y & A D_{16} A D_{\mathrm{g}} & B I \\
\overline{P A B A} & \bar{Y} & a d_{16} & a d_{\mathrm{B}} & b i\end{array}$ & $\mathbf{I}$ \\
\hline iv & wild type & $\begin{array}{lll}p a b a & y \\
P A B A & Y A D_{16} A D_{\mathrm{B}} & b i \\
a d_{16} A D_{\mathrm{B}} & B I\end{array}$ & 2 \\
\hline $\mathbf{v}$ & wild type & $\begin{array}{lllll}p a b a & y A D_{16} A D_{8} & B I \\
P A B A & Y a d_{16} A D_{8} & b i\end{array}$ & 5 \\
\hline vi & biotin-requiring & 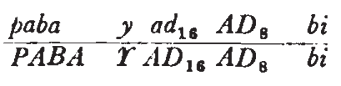 & 1 \\
\hline vii & wild type & $\begin{array}{lllll}p a b a & y & a d_{16} A D_{8} & B I \\
P A B A & Y & A D_{16} A D_{8} & b i\end{array}$ & 1 \\
\hline viii & wild type & $\begin{array}{lll}p a b a & y A D_{16} a d_{8} & B I \\
P A B A & Y A D_{16} A D_{8} & b i\end{array}$ & 4 \\
\hline ix & biotin-requiring & $\frac{p a b a}{P A B A}-\frac{Y A D_{16} A D_{\mathrm{B}}}{Y} \frac{b i}{a d_{16} A D_{\mathrm{B}}} \quad b i$ & I \\
\hline $\mathbf{x}$ & wild type & 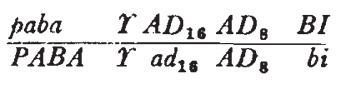 & 1 \\
\hline xi & wild type & $\begin{array}{lll}p a b a & y A D_{16} A D_{\mathrm{8}} & B I \\
P A B A & \Gamma A D_{16} A D_{\mathrm{8}} & b i\end{array}$ & I \\
\hline xii & yellow & Not analysed & I \\
\hline xiii & $\begin{array}{l}\text { yellow, P.A.B.A., } \\
\text { pyridoxin }\end{array}$ & Not analysed & 1 \\
\hline
\end{tabular}

* All were heterozygous for W/w and NIC/nic, or for PYRO/pyro except type xiii.

Classes III, IV and v.-Diploids in each of these classes have genotypes expected following the simultaneous occurrence of two mitotic exchanges, one between the alleles and a second between $a d_{8}$ and $b i$. No other simple explanation can account for the genotype of Classes iii and iv, but Class $v$ diploids would also be produced following back-mutation of $a d_{8}$. In fig. 3 the eight possible types that can be produced following a two-, three- or four-strand double exchange of this type are indicated. 
The Class iii diploid requires a two-strand double exchange and inclusion of both crossover products in the same daughter nucleus. The presence of $a d_{16}$ on the green strand in coupling with $a d_{8}$ was verified by outcrossing in the manner already described.

The two Class iv diploids can only be produced following one type of three-strand double, or a four-strand double. On the assumption that the four types of double exchange occur with equal frequency

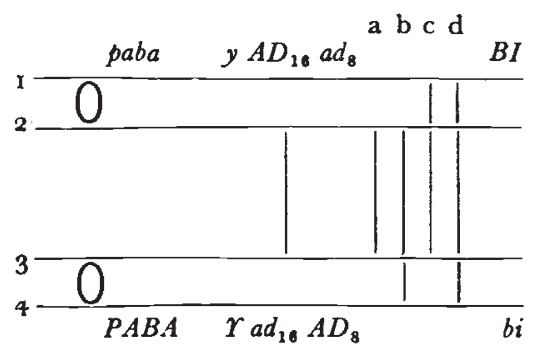

\begin{tabular}{|c|c|c|c|c|}
\hline $\begin{array}{l}\text { Type of } \\
\text { double }\end{array}$ & $\begin{array}{c}\text { Chromatids } 2 \text { and } 3 \text { to } \\
\text { daughter nucleus }\end{array}$ & Class & $\begin{array}{c}\text { Chromatids } 2 \text { and } 4 \text { to } \\
\text { daughter nucleus }\end{array}$ & Class \\
\hline a & $\begin{array}{lcc}p a b a & y A D_{16} A D_{8} B I \\
P A B A & \Upsilon a d_{10} a d_{8} & b i\end{array}$ & iii & $\begin{array}{ccc}p a b a & y A D_{16} A D_{8} & B I \\
P A B A & \Upsilon a d_{10} A D_{8} & b i\end{array}$ & $\mathrm{v}$ \\
\hline b & $\begin{array}{lcc}p a b a & y A D_{16} A D_{8} & b i \\
\overline{P A B A} & \Upsilon_{a d_{16} a d_{8}} \quad b i\end{array}$ & & $\begin{array}{lll}p a b a & \text { y } A D_{16} A D_{8} & b i \\
P A B A & \operatorname{rad}_{16} A D_{8} & B I\end{array}$ & iv \\
\hline c & $\frac{p a b a}{P \overline{A B A}} \frac{y A D_{16} A D_{8} B I}{\Upsilon \operatorname{ld}_{16} a d_{8}} \quad B I$ & & $\begin{array}{ccc}p a b a & y A D_{16} A D_{8} & B I \\
P A B A & \Upsilon_{a d_{18}} A D_{8} & b i\end{array}$ & $v$ \\
\hline d & $\frac{p a b a}{\overline{P A B A}} \frac{y}{\Upsilon a d_{16} a d_{8}} \quad B I$ & & $\frac{p a b a}{P A B A} \frac{y A D_{16} A D_{8}}{\operatorname{rad}_{16} A D_{8}} \quad b i$ & iv \\
\hline
\end{tabular}

Fig. 3.-The 8 possible types of adenine-independent diploid produced by two mitotic exchanges, one between $a d_{16}$ and $a d_{8}$ and a second between $a d_{8}$ and $b i$. Daughter nuclei carrying chromatid $I$ are adenine-requiring and are not shown.

and segregation of the pairs of chromatids is random, diploids Class iv are expected to arise twice as frequently as diploids Class iii. The actual numbers are 2 and $\mathrm{I}$.

The five Class $\mathrm{v}$ diploids require a two- or three-strand double exchange and repeating the same assumptions would be expected to occur with a frequency equal to that of Class iv. They could also occur as a result of back-mutation of $a d_{8}$ since they have a genotype identical with that of the parent except that $a d_{8}$ has been replaced by its wild type allele. There is no way of distinguishing between these two alternatives but the fact that back-mutation of $a d_{8}$ had not been previously encountered, and that diploids of this type produced by a double mitotic exchange were expected with a frequency at least equal to that of Class iv diploids, suggested that back-mutation was the least likely of the two alternatives. 
The occurrence of possible back-mutants made it desirable to make a test for back-mutation of $a d_{8}$ in a diploid homozygous for this mutant. From such a diploid $3.42 \times 10^{8}$ conidia were tested and 6 reversions were obtained, of which 5 were due to back-mutation of $a d_{8}$ on the green strand and I on the yellow strand (table ro). The first 5 may have been repeated isolates from a single clone, but the results indicate that at least 2 back-mutants were picked up. The origin of the Class v diploids cannot therefore be decided.

ClaAsses vi and vir.-The occurrence of these two diploids was extremely significant and their origin must be considered at some length. Both carried a strand with $y$ and $a d_{16}$ in coupling whereas in the parent diploids these were in repulsion. This can only be

TABLE 10

The genotype of adenine-independent mitotic segregants obtained from two diploids, one homozygous for $\mathrm{ad}_{8}$ and the other for $\mathrm{ad}_{16}$

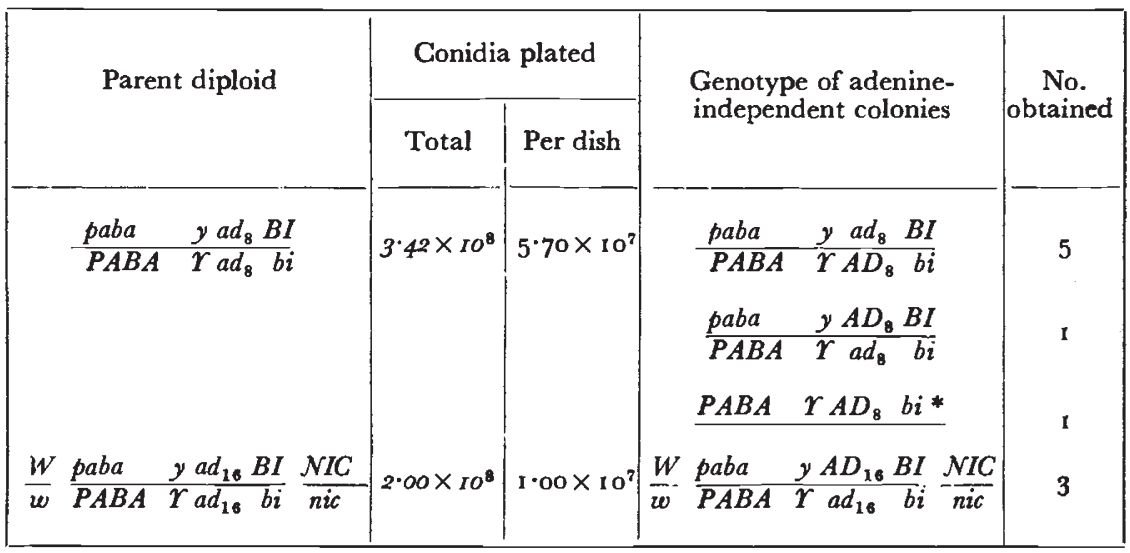

* This haploid could be a contaminant or represent a case of back-mutation followed by haploidisation.

satisfactorily accounted for it there has been an exchange between $y$ and $a d_{16}$. As a result of this exchange the yellow strand should carry $b i$ and this is found to be the case for diploid vi, but on the contrary diploid vii carries $B I$ on this strand. Unless the extremely improbable assumption is made that back-mutation of $b i$ has occurred, a second exchange must have occurred between $a d_{8}$ and $b i$ in this diploid. The green strand of both diploids carries the parental markers except that $a d_{16}$ has been replaced by its wild type allele. There are two possibilities that will account for this. Either back-mutation of $a d_{16}$ has occurred or a further mitotic exchange between the alleles.

If the former assumption is made then back-mutation of $a d_{16}$ has on two occasions been accompanied by a mitotic exchange between $y$ and $a d_{16}$. This can scarcely be due to coincidence since the recombination fraction between these two loci is not greater than 0.002 .

The results of meiotic analysis of crosses between alleles indicated that reversion to adenine-independence was associated with crossing 
over between $y$ and $b i$. It was an extrapolation from the data, not necessarily correct, that the exchange occurred between the alleles. It might be argued that an exchange close to the right or left of the alleles might also result in an adenine-independent strand. If this were true it might be argued that the exchange between $y$ and $a d_{16}$ in diploids vi and vii, and the reversion of $a d_{16}$ are but two aspects of one event. Such an assumption is still unable to explain the genotype of diploids vi and vii, however, since the strand in which
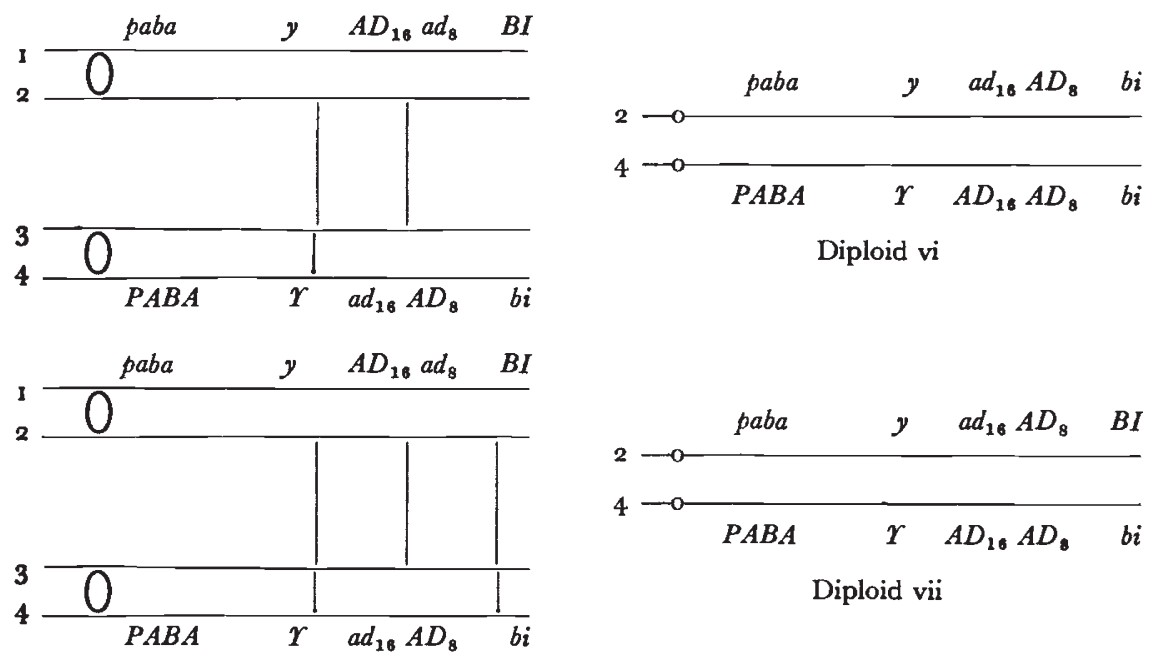

Diploid vii
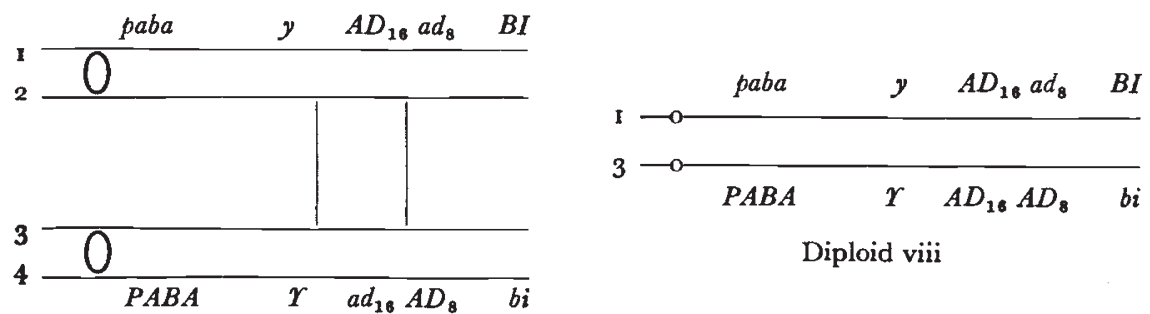

Diploid viii

Fig. 4.-Types of mitotic exchange which may produce diploids of classes vi, vii and viii. The parent diploid is shown on the left.

reversion of $a d_{16}$ to $A D_{16}$ could have occurred cannot be either of those involved in the exchange between $y$ and $a d_{16}$. One would have to assume that an exchange between two strands results in reversion of $a d_{16}$ on a third.

On the other hand, if it is assumed that an exchange between the two alleles greatly increases the probability of a second exchange nearby, as is strongly suggested by the results of meiosis in crosses involving pairs of alleles, then the genotypes of diploids vi and vii offer no difficulty. In vi an exchange between the alleles has been accompanied by a second exchange between $y$ and $a d_{16}$, as indicated in fig. 4 , and in vii an exchange between $a d_{8}$ and $b i$ has occurred in addition (fig. 4). 
Since the conclusion that multiple exchanges have occurred to give rise to vi and vii is based on the fact that diploids of both these classes possess a strand with $y$ and $a d_{16}$ in coupling, it should be pointed out that these diploids could not be contaminants. No strain, haploid or diploid, with these two mutants in coupling was in existence when these diploids were recovered. Moreover, vi was obtained from diploid II and was heterozygous $W / w$ and $\mathcal{N I C / n i c}$ and vii, from diploid I, was heterozygous PYRO/pyro.

Class virI.-The four diploids in this class are identi al with the parent diploids except that $a d_{16}$ has been replaced bv its wild type allele. The absence of $a d_{16}$ from the yellow strand was in each case verified by outcrossing to an adenine-independent strain. Backmutation of $a d_{16}$ or certain types of double mitotic exchange between $y, a d_{16}$ and $a d_{8}$ (see fig. 4) will both produce diploids of this type. Since Class vi and vii diploids indicate that double exchanges of this type do occur, and since back-mutants of $a d_{16}$ were obtained from a homozygote $a d_{18} / a d_{16}$ (table 1o), the origin of these diploids cannot be determined.

CLASSES IX AND X.-These two diploids were similar in that both were homozygous $Y / \Upsilon$. Back-mutation of $y$ to $r$ can be rejected as extremely improbable. There must therefore have been a mitotic exchange between paba and $y$ followed by segregation of one crossover and one non-crossover strand to the daughter (fig. 5). If there had been no further exchanges, both diploids would be homozygous $b i / b i$. Diploid xi, however, is heterozygous $B I / b i$ and a further exchange must have taken place between $y$ and $b i$ unless mutation is again invoked. Both are, of course, adenine-independent. Either backmutation of $a d_{16}$ or two further mitotic exchanges are required.

Thus two explanations to account for diploid $\mathbf{x}$ are possible. Either back-mutation of $a d_{16}$ has been accompanied by a mitotic exchange between $p a b a$ and $y$, or three mitotic exchanges have occurred simultaneously. Diploid xi similarly requires either back-mutation of $a d_{18}$ together with two mitotic exchanges, or four mitotic exchanges.

The occurrence of an exchange between paba and $y$ simultaneously with or subsequent to back-mutation of $a d_{16}$ in two diploids out of $4 \mathrm{I}$ analysed can scarcely be due to chance, since the former event occurs with very low frequency. Thus from a plating of approximately 500 conidia from both parent diploids no yellow colonies were obtained, although one-quarter of the mitotic exchanges between $y$ and the centromere would result in colonies of this type. On the other hand, it is difficult to understand the nature of an association between mutation and crossing over certainly at a different locus (the locus $y$ intervenes between the sites of mutation and crossing over). The latter of the two alternatives would therefore seem the more reasonable.

CLASS XII.-The extraordinary aspect of the genotype of this diploid was that it possessed no ad allele at all. Either simultaneous back-mutation of both $a d_{16}$ and $a d_{8}$ are required or four mitotic 
exchanges, two of which must be between the alleles (fig. 5). It is unlikely that this diploid was a contaminant since it was heterozygous PYRO/pyro.

\section{(iv) Discussion}

Before discussing the significance of the results of mitotic analysis, it should be recalled (Pontecorvo et al., I 954 and personal communication) that mitotic crossing over occurs with low frequency and that coincidence of two, let alone more, mitotic exchanges in the arm of the chromosome studied here is a rare event.
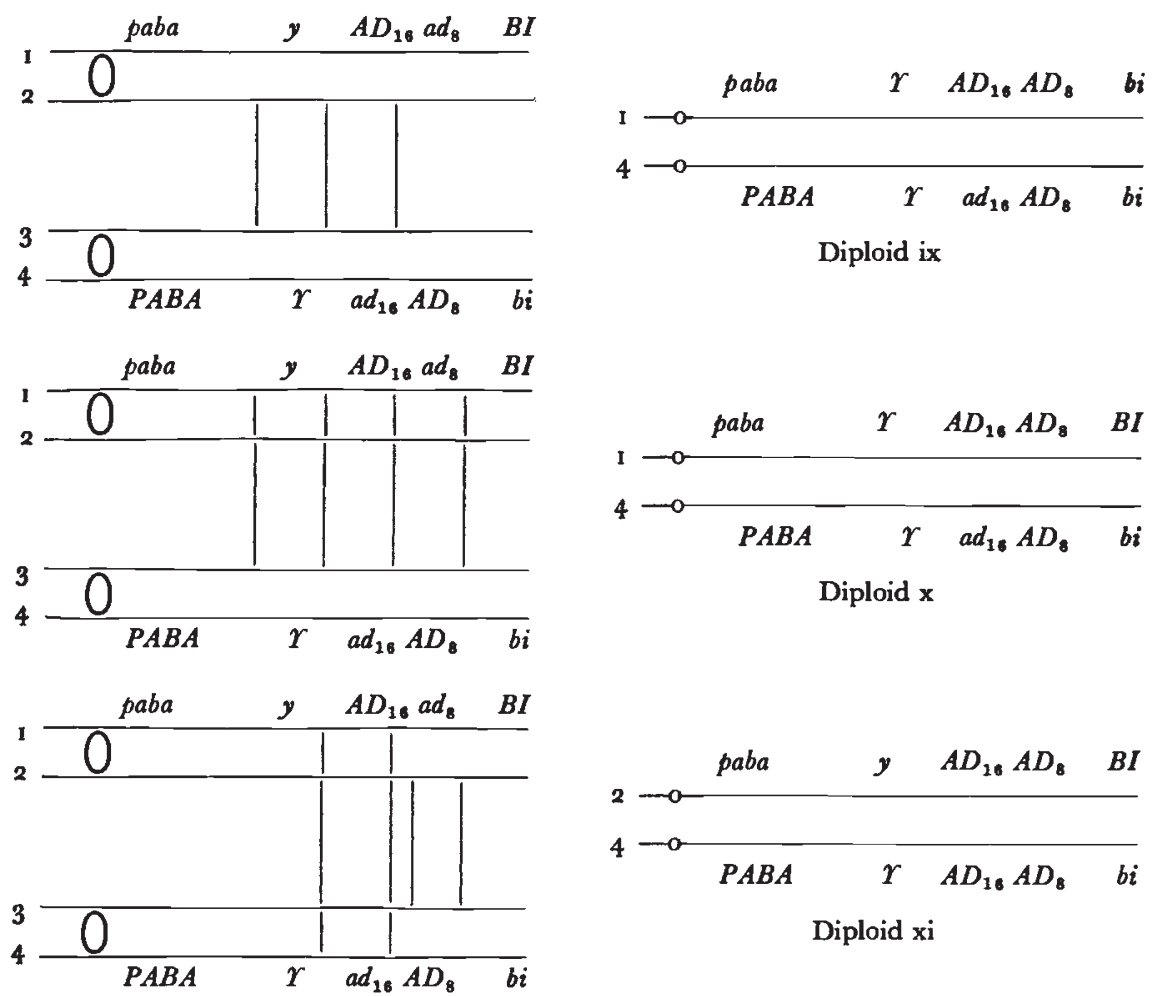

FIG. 5.-Types of mitotic exchange which may produce diploids of classes $\mathrm{ix}, \mathrm{x}$ and $\mathrm{xi}$. Only one of several possible types of strand arrangement is shown.

Mitotic analysis has shown that there is an unquestionable association between reversion to adenine-independence and crossing over between $y$ and $a d_{16}$, or between $a d_{8}$ and $b i$. In most cases it was also clear that the change from adenine-dependence to independence was due to or associated with crossing over between the alleles, but in some cases the results could also be interpreted as due to the simultaneous occurrence of back-mutation of one or other allele and mitotic crossing over elsewhere. The ambiguity of these types was due to the fact that if multiple exchanges indeed occurred, they were 3- or 4-strand doubles, or multiples, in which more than two strands were involved. The reciprocal products in multiples of this type 
cannot be recovered mitotically by our technique, though they could by tetrad analysis in meiosis. Alternatively the non-reciprocal products of a 2-strand multiple were recovered.

Coincidence of back-mutation of $a d_{8}$ or $a d_{16}$ with mitotic crossing over is unlikely owing to the rarity of the two events. On the other hand, an association between mutation and crossing over cannot be ruled out, particularly in view of the recent work of Mitchell (1955) with Neurospora. While we have no evidence at present that would favour an interpretation of this type there is positive evidence of the occurrence of additional mitotic exchanges following an exchange between alleles, as for example in Classes iii and iv. It therefore seems reasonable at present to conclude that possible cases of mutation associated with mitotic crossing over are instances of multiple mitotic crossing over in which, for the reasons already stated, both products of every exchange have not been recovered.

If the results of mitotic analysis have been interpreted correctly a multiple mitotic exchange rate greater than expected at meiosis has indeed been observed. Thus types iii, iv, vii, $x$ and $x i$, a total of 6 , all have an exchange between $a d_{8}$ and $b i$ in addition to one between the alleles. The fraction of doubles is $6 / 36$ or $16 \cdot 7$ per cent. (the 5 Class $v$ diploids are excluded from this calculation since they are either back-mutants or doubles of the type considered here). Even if types vii and $\mathbf{x}$ are excluded as possible cases of backmutation associated with crossing over and class xi as a case of simultaneous back-mutation of both $a d_{8}$ and $a d_{16}$, the frequency of doubles (9.I per cent.) remains not only enormously higher than expected from chance coincidence of two mitotic exchanges, but also higher than expected at meiosis.

Similarly there were at least two (vi and vii), and possibly three more (ix, $x$ and $x i$ ), diploids in which crossing over had taken place between $y$ and $a d_{16}$ although these loci are extremely closely linked (see table I).

It is clear from the mitotic data that inversions cannot be responsible for the excess of multiple exchanges observed since the majority of these are not 2-strand doubles.

\section{GENERAL DISCUSSION}

Two questions are considered in this section : the bearing of the results obtained here on the problem of allelism, and the meaning of the intense negative interference discovered.

The crosses involving pairs of alleles in what will be called the " $a d_{8}$ region" identify at least four mutational sites separable by crossing over and arranged in the linear order : $a d_{16}, a d_{11}, a d_{8}$ and $a d_{10}$. The data also indicate that $a d_{12}, a d_{19}$ and $a d_{20}$ are mutants of different sites from $a d_{8}$ and $a d_{10}$ although their relationships to each other and to $a d_{16}$ and $a d_{11}$ have not yet been worked out. The recombination fractions between ad alleles and between $y$ and different 
ad alleles are for the most part consistent with each other (see fig. 6, which gives all recombination fractions so far measured).

With the exception of the cross involving $a d_{8}$ and $a d_{10}$ there is no evidence from either meiotic or mitotic analysis that unequal crossing over is involved in the origin of adenine-independent types, and even in this cross the results may be due to multiple exchanges in view of the results of mitotic analysis.

Infertility has so far prevented the test of all the available alleles in the $a d_{8}$ region against each other. This would have provided an estimate of the probable number of sites of mutation separable by crossing over in this region. But other evidence from $A$. nidulans alone already suggests that the frequency of independent re-occurrences of the "same" mutation, i.e. not distinguishable from another one by a test of crossing over, is small, and consequently that the number of

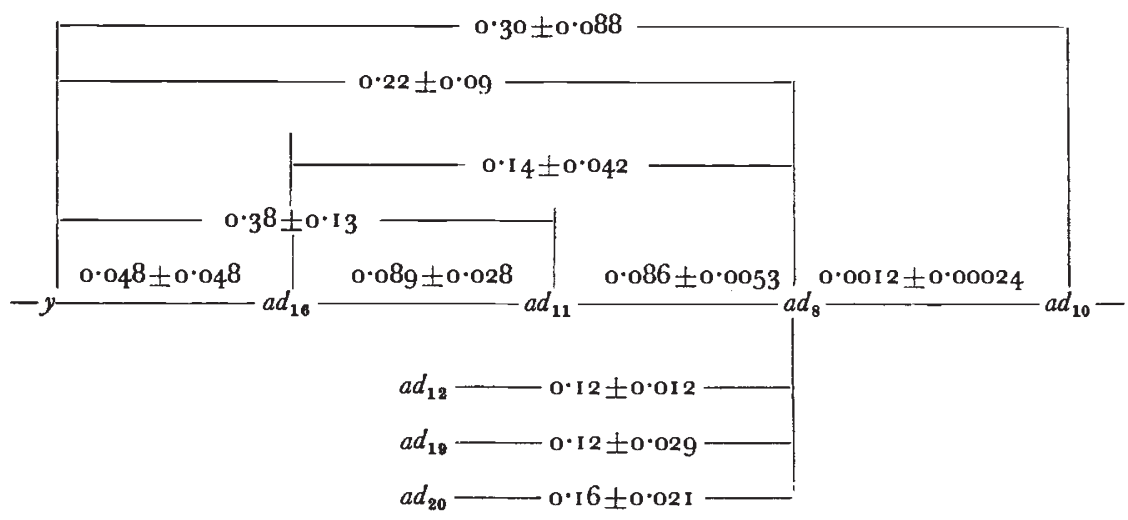

FIG. 6.-Linkage map of the $a d_{8}$ region giving all recombination fractions $\left(\times \mathrm{IO}^{2}\right)$ so far measured.

sites of mutation within chromosome segments behaving as functional units is large. Thus, tests between over twenty alleles in five regions have in no case failed to yield recombinants in experiments with a resolving power of $\mathrm{IO}^{-5}$ (Roper, I953 ; Pontecorvo, I955 ; Calef, unpublished ; Forbes, unpublished).

Explanations of the Lewis effect in terms of sequences of reactions and localised gene products therefore seem less satisfactory than at first supposed by Pontecorvo (I950, I 952a, I952b). On the other hand, the occurrence of suppressors specific for some but not others of an allelic series (Giles and Partridge, I953; Yanofsky, I953; Green 1954)-and $a d_{20}$ is a further example-and the separation of "suppressible" and " non-suppressible" alleles by crossing over, has been considered evidence that the two types of allele are mutants of " different genes with distinctive loci and functions" (Green, I 954). It is equally reasonable to suppose, however, that mutation at different sites, or different types of mutation at one site, within a single functional unit can result in qualitatively or quantitatively distinct modifications of a single normal gene product. Some types of modification, probably 
the less extreme, might give rise to a mutant phenotype under certain intracellular conditions but not in others. A change of $p \mathrm{H}$, for example, produced by a suppressor mutation might suppress a mutant which produced an enzyme with an altered $p \mathrm{H}$ optimum, but not one which completely prevented the synthesis of the enzyme.

Clearly distinction between alternative explanations of the Lewis effect must await more critical information. In particular, we need more information as to the probable number of sites of mutation within chromosome segments behaving physiologically as a unit, and as to qualitative differences between alleles.

The occurrence of negative interference associated with recombination between ad alleles raises a number of questions. How far does the influence of a crossover between alleles extend? Is negative

TABLE II

Recombination fractions between paba and $\mathrm{y}, \mathrm{y}$ and $\mathrm{ad}$, and $\mathrm{ad}$ and bi calculated only among crossovers between ad alleles (from the data of tables 2, 5, 6 and 7 )

\begin{tabular}{|c|c|c|c|c|}
\hline $\begin{array}{l}\text { ad alleles } \\
\text { involved in } \\
\text { the cross }\end{array}$ & $\begin{array}{c}\text { Recombination } \\
\text { fraction between } \\
\text { paba and } y\end{array}$ & $\begin{array}{l}\text { Probability } \\
\text { of deviation } \\
\text { from } 0 \cdot 15\end{array}$ & $\begin{array}{c}\text { Recombination } \\
\text { fraction between } \\
y \text { and ad }\end{array}$ & $\begin{array}{c}\text { Recombination } \\
\text { fraction between } \\
\qquad a d \text { and } b i\end{array}$ \\
\hline $\begin{array}{l}8 \text { and } 10 \\
8 \text { and } 11 \\
8 \text { and } 12 \\
8 \text { and } 16 \\
8 \text { and } 19 \\
8 \text { and } 20 \\
11 \text { and } 16\end{array}$ & $\begin{aligned} 2 / 28 & =0.071 \\
28 / 179 & =0.16 \\
25 / 139 & =0.18 \\
25 / 139 & =0.18 \\
6 / 28 & =0.21 \\
28 / 79 & =0.35 \\
6 / 57 & =0.10\end{aligned}$ & $\begin{array}{l}\text { not sig. } \\
\text { not sig. } \\
\text { not sig. } \\
\text { not sig. } \\
\text { not sig. } \\
0 \cdot 001 \\
\text { not sig. }\end{array}$ & $\begin{aligned} 6 / 28 & =0.21 \\
10 / 365 & =0.027 \\
6 / 139 & =0.043 \\
6 / 139 & =0.043 \\
1 / 28 & =0.036 \\
8 / 79 & =0.10 \\
11 / 57 & =0.19\end{aligned}$ & $\begin{array}{l}11 / 28=0.39 \\
43 / 179=0.24 \\
32 / 139=0.23 \\
31 / 139=0.22 \\
10 / 28=0.36 \\
13 / 79=0.16 \\
6 / 57=0.10\end{array}$ \\
\hline
\end{tabular}

interference associated only with crossing over between alleles or between any closely linked mutants? Is it a general property of recombination between alleles or closely linked mutants in all organisms or is it confined to $A$. nidulans or even to the $a d_{8}$ region.

The recombination fraction between $p a b a$ and $y$ among crossovers between ad alleles from all available crosses has been calculated in table I I. On the same table these values are compared with $0 \cdot I_{5}$, the standard recombination fraction between these two loci. In five of these crosses this fraction among crossovers was greater than $0 \cdot 15$ (significantly so only in one). There is therefore some indication that negative interference associated with an exchange between alleles extends beyond $y$.

The data in table I I also suggest that the degree of negative interference associated with recombination between alleles is different in different crosses and shows no apparent correlation with the position in the $a d_{8}$ region of the alleles involved. It has been assumed in the table, however, that all $A D$ types showing no recombination for $y$ and $b i$ were double exchanges with one exchange between two alleles and a second between $y$ and $b i$. The possibility cannot of course be excluded that some have originated by mutation. 
In order to answer the question of the dependence of negative interference on crossing over between any two closely linked loci, all available data from crosses involving different ad mutants and an adenine-independent strain were re-examined (table 12). The recombination fraction between an ad mutant and $b i$ (or between $y$ and $b i$ which will have approximately the same value) can be compared with the same fraction among crossovers between the ad mutant and $y$. The number of crossovers obtained between any one ad mutant and $y$ is too small to give statistically significant information, but the results as a whole strongly suggest that exchanges between $y$ and an $a d$ mutant also increase the probability of a second exchange nearby, i.e. between ad and $b i$.

A similar comparison of the recombination fraction between paba and $y$ among crossovers and non-crossovers between $y$ and different ad mutants (table I2) gives little evidence of negative interference, but in view of the distance between paba and $y$ much more extensive data would be needed for significant information.

In connection with the question of how general the properties described here are, there are a number of important observations. In Aspergillus, similar effects are associated with recombination between alleles of paba (Roper, unpublished), and with recombination between closely linked mutants of the $a d_{9}$ region (Calef, unpublished). In Neurospora, the results obtained by Giles (1951) from a cross between two inositol alleles showing recombination were strikingly similar to those obtained here. The frequency of inositol independent cultures with parental combinations of markers was considerably greater than expected from the known distance between these markers, but platings of ascospores from crosses between strains carrying the same inositol allele indicated that the results could probably not be attributed to back-mutation. The results of Weijer (1954) from crosses involving allelic tryptophan (" $t d$ ") mutants suggest that negative interference is associated with recombination between alleles here as well, although less reliance can be placed on these results since markers were available on one side of the " $t d$ " region only.

In Drosophila on the other hand, published data on crossing over between very closely linked mutants give no evidence of negative interference. On the contrary, the results of Green and Green (1949) and Lewis (1945) suggest that crossing over between alleles is associated with positive interference. Certain so-called mutations of "Bar" (Sturtevant, I925 ; Bonnier, Nordenskiōld and Bågman, 1943) could have been produced by multiple crossing over within a very short chromosome segment, but unequal sister-strand crossing over would also produce the same types. It was suggested to me by Prof. C. D. Darlington that "reciprocal crossing over" in the sex chromosomes of Drosophila (Darlington, I934) might be an evolutionary exploitation and modification of a system of negative interference of the type encountered here. 


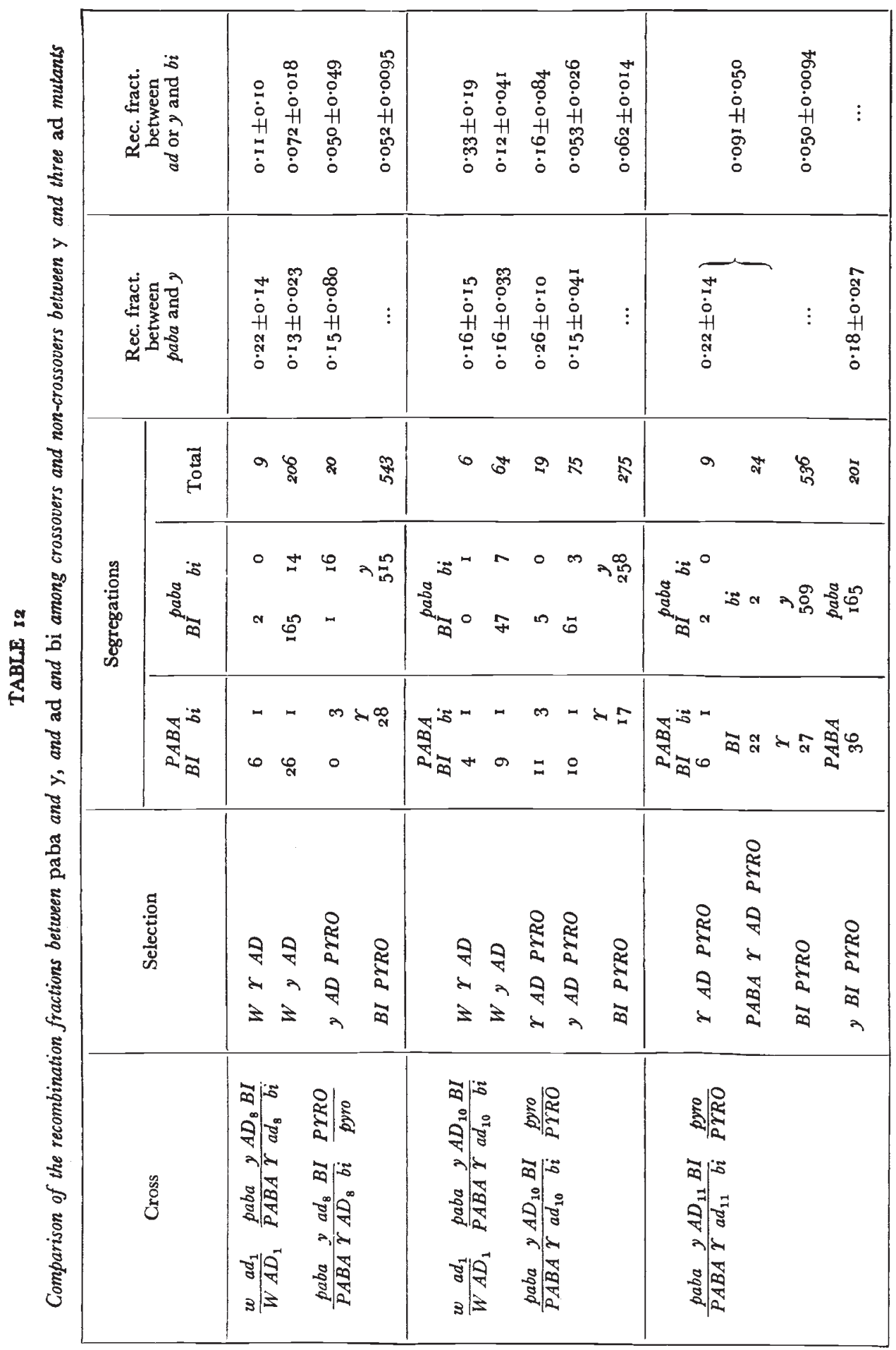


Any model to account for negative interference of the type found here must take into account that negative interference is not observed when recombination between loosely linked mutants is followed (extensive data from many crosses in $A$. nidulans involving paba, $y$ and $b i$ give no evidence of interference, either positive or negative). One possibility is that " effective" pairing (that is pairing that can lead to crossing over-not necessarily identical with pairing observed cytologically) is confined to short chromosome segments, at any one point the homologues usually remaining "effectively" unpaired. Positive interference would occur if " effective " pairing of one segment reduced the probability of "effective" pairing of neighbouring segments. Within "effectively" paired segments, however, negative interference would be apparent (Rothfels, 1952).

If a model of this type is correct an important question is the length of "effectively" paired segments. The association of negative interference with recombination between ad alleles would not be of fundamental importance if the apparent close linkage between the $a d_{8}$ region and $y$ were simply due to suppression of crossing over by pairing failure. On the other hand if the chromosome segment between the $a d_{8}$ region and $y$ is of the same order of length as the $a d_{8}$ region itself, as the map distances suggest, then multiple crossing over within a chromosome segment of the same size as that occupied by one gene has been observed. In Aspergillus, where cytological localisation of mutants is not possible, these alternatives cannot be distinguished until the segregation of three alleles has been followed simultaneously, but the latter alternative raises the possibility that single crossovers detected either cytologically or genetically are frequently the net result of two or more exchanges so close together as to be detectable only by the use of extremely closely linked mutants, or possibly in tetrad analysis as an excess of non-parental ditypes. The ease with which multiple exchanges of this type could be detected would depend on the length of "effective" pairing segments. Variation of this might account for the difference between Drosophila and Aspergillus with respect to the occurrence of negative interference.

Present information does not warrant the building of a precise working model for the basis of negative interference and of its connections with crossing over and chromosome re-duplication. The data so far give only a clear indication that analysis of crossing over within very short chromosome segments may reveal novel modalities.

\section{SUMMARY}

r. The first four out of a group of nine physiologically allelic adenine-requiring mutants of Aspexgillus nidulans were found to be mutants at different, but very closely linked, loci separable by crossing over. Three others were found to be mutants at loci different from two of the first four, but their location with respect to the other two and to each other has not been determined. 
2. The results provide evidence additional to that already available in four other chromosome regions of the species and from other organisms, that physiologically allelic mutants are not usually the result of re-occurrence of mutation at the same locus. Within a chromosome segment behaving functionally as a unit there are several, probably very many, sites capable of independent mutation and likewise several sites of crossing over.

3. Two suppressors specific for one but not others of these alleles have been found.

4. Meiotic analysis suggested that a crossover between alleles, and perhaps between any very closely linked mutants, is associated with negative interference. Analysis of half-tetrads following mitotic crossing over has provided further evidence to support this.

5. There is evidence that negative interference of this type occurs in other organisms. This raises the possibility that single crossovers detected cytologically or genetically may be the net result of clusters of exchanges so close together as to be detectable only under special conditions.

Acknowledgments.-The receipt of a maintenance grant from the Department of Scientific and Industrial Research is gratefully acknowledged. This work is part of a programme supported by the Nuffield Foundation.

For his inspiration and guidance, my sincere thanks are due to Dr G. Pontecorvo.

\section{REFERENCES}

BONNIER, G., NORDENSKIŌLd, M., AND BÅGMAN, G. I943. Exaggeration of Bar in Drosophila melanogaster. Hereditas, 29, i 1 3-133.

DARLington, c. D. 1934. Anomalous chromosome pairing in the male Drosophila pseudo-obscura. Genetics, 19, 95-1 18.

GILES, N. H. I95I. Studies on the mechanism of reversion in biochemical mutants of Neurospora crassa. Cold Spr. Harb. Symp. Quant. Biol., 16, 283-313.

Giles, N. H., AND PARTRIDGe, c. W. H. I953. The effect of a suppressor on allelic inositolless mutants in Neurospora crassa. P.N.A.S., 39, 479-488.

Green, M. M. 1954. Pseudoallelism at the vermilion locus in Drosophila melanogaster. P.N.A.S., 40, 92-99.

GREEN, M. M., AND GREEN, K. c. 1949. Crossing over between alleles at the lozenge locus in Drosophila melanogaster. P.N.A.S., 35, 586-591.

GRIGG, G. W. 1952. Back mutation assay method in micro-organisms. Nature. 169, 98-1 оo.

haldane, J. B. S. 1954. The Biochemistry of Genetics. London : Allen and Unwin. LEWIS, E. B. 1945. The relation of repeats to position effect in Drosophila melanogaster. Genetics, 30, $137-166$.

LEwis, E. B. 1951. Pseudoallelism and gene evolution. Cold Spr. Harb. Symp. Quant. Biol., 16, I59-1 74 .

LURIA, S. E., AND DELBRUCK, M. I943. Mutations of bacteria from virus sensitivity to virus resistance. Genetics, 28, 49 I-5I I.

macdonald, K. D., AND pontecorvo, G. 1953. The genetics of Aspergillus nidulans. Adv. Genet., 5, 159-1 70.

MITCHELL, M. M. I955. Aberrant recombination of pyridoxin mutants of Neurospora. P.N.A.S., 4I, 21 5-220.

MULLER, H. J. 1947. The gene. P.R.S., B, 134, I-37.

PONTECORvo, G. 1950. New fields in the biochemical genetics of microorganisms. Biochem. Soc. Symp., 4, 40-50. 
PONTECORvo, G. 1952a. The genetical formulation of gene structure and action. Adv. Enzymol., 13, 12 1-149.

POntecorvo, G. 1952b. Genetical analysis of cell organisation. Symp. Soc. exp. Biol., 6, 2 18-229.

Pontecorvo, G. 1953. The genetics of Aspergillus nidulans. Adv. Genet., 5, 141-238. PONTECORvo, G. 1954. Alleles or pseudoalleles. Heredity, 8, 434 (abstract).

PONTECORvo, G. 1955. Gene structure and action in relation to heterosis. P.R.S., $B, 144,171-177$.

PONTECORVO, G., AND KAFER, E. I954. Maps of a chromosome region in Aspergillus nidulans based on mitotic and meiotic crossing over. Heredity, 8, 433 (Abstract).

PONTECORVO, G., TARR GLOOR, E., AND FORBES, E. 1954. Analysis of mitotic recombination in Aspergillus nidulans. 7. Genet., 52, 226-237.

pontecorvo, G., AND ROPER, J. A. 1953. The genetics of Aspergillus nidulans. Adv. Genet., 5, $218-233$.

PONTECORVO, G., ROPER, J. A., AND FORBES, E. I953. Genetic recombination without sexual reproduction in Aspergillus niger. 7. gen. Microbiol., 8, 198-2 10.

PRITCHARD, R. H. 1953. Ascospores with diploid nuclei in Aspergilltes nidulans. Proc. gth Int. Cong. Genet. (in the press).

PRITCHARD, R. H. 1954. The relationship between a group of alleles in the $a_{8}$ region of Aspergillus nidulans. Heredity, 8, 433 (Abstract).

PRITCHARD, R. H., AND PONTECORVO, G. I 953 . The formation of ascospores with diploid nuclei in Aspergillus nidulans. Microb. Genet. Bull., 7, 18.

RAFFel, D., AND MUller, H. J. 1940. Position effect and gene divisibility considered in connection with three strikingly similar scute mutations. Genetics, 25, 541-583.

ROPER, J. A. 1952. Production of heterozygous diploids in filamentous fungi. Experientia, 8, 14-15.

ROPER, J. A. I953. The genetics of Aspergillus nidulans. Adv. Genet., 5, 208-215.

ROPER, J. A., AND PRITCHARD, R. H. 1955. The recovery of the complementary products of mitotic crossing over. Nature, 175, 639 .

ROTHFELS, K. H. 1952. Gene linearity and negative interference in crosses of Escherischia coli. Genetics, 37, 297-311.

Sturtevant, A. H. 1925. The effects of unequal crossing over at the Bar locus in Drosophila melanogaster. Genetics, 10, I 1 7-147.

WEIJER, J. 1954. A genetical investigation into the $t d$ locus of Neurospora. Genetica, 27, $173-252$.

YANOFSKY, C. 1953. Further studies with the $t d$ mutants of Neurospora crassa. Genetics, 38, 702 (abstract). 